\title{
El uso de bases de datos curatoriales para reconstruir la historia del conocimiento taxonómico: un ejemplo con papiliónidas y piéridas mexicanas (Insecta: Lepidoptera)
}

\author{
Use of curatorial data bases as an element to reconstruct taxonomic knowdlege: an example \\ with Mexican papilionids and pierids (Insecta: Lepidoptera)
}

Leonor Oñate-Ocaña y Jorge Llorente-Bousquets*

Museo de Zoología, Facultad de Ciencias, Universidad Nacional Aautónoma de México. Apartado postal 70-399, 04510 México, D.F., México. *Correspondencia:jlb@hp.fciencias.unam.mx

\begin{abstract}
Resumen. El objetivo de este trabajo es conocer la cronología de la información sobre mariposas papiliónidas y piéridas de México, así como los patrones de recolección y los recolectores más destacados a partir de una base de datos curatoriales y fuentes bibliográficas. La base cuenta con 40752 registros de ejemplares albergados en 12 museos y/o registrados en 145 fuentes bibliográficas. Se destaca el alcance que tiene el uso de las bases de datos curatoriales en la reconstrucción histórica del conocimiento de un grupo, tomando como ejemplo las mariposas papilónidas y piéridas mexicanas.

Palabras clave: mariposas, ropalóceros, México, colecciones biológicas.

Abstract. The aim of this paper is to determine the chronology of the information on papilionid and pierid butterflies of Mexico, as well as the patterns of collection and who were the most active collectors from a curatorial databases and bibliography. The database has record of 40752 records of individuals housed in 12 museums and/or recorded in 145 bibliographic sources. The relevance of this work is to highlight the amplitude of curatorial databases for the reconstruction of the historical knowledge of a biological group, taking Mexican papilionid and pierid butterflies as an example.
\end{abstract}

Key words: butterflies, Rhopalocera, Mexico, biological collections

\section{Introducción}

En los últimos 20 años se ha incrementado el uso de las bases de datos curatoriales para estimar la riqueza de especies y los cambios en la biodiversidad, así como otros aspectos de análisis espacial (Peláez, 1994; Soberón et al., 2000; Martín-Piera y Lobo, 2003). Las bases de datos curatoriales también contienen información cronológica que puede utilizarse para reconstruir la historia del conocimiento de la biodiversidad. La mayor parte de esa información se obtiene de colecciones biológicas (Navarro y Llorente, 1994; Navarro et al., 2003a, b) que contienen los datos del colector, el sitio, la fecha de de la recolección, y otros datos que no se encuentran en los registros de especies descritas en la bibliografía. Es por esto que los museos se convierten en centros privilegiados para la investigación y planeación de estrategias de conservación (Peláez, 1994; Navarro y Llorente, 1994; Peterson et al., 1998; Navarro et al., 2003a) y las bases de datos procedentes de esas fuentes

Recibido: 24 octubre 2008; aceptado: 19 octubre 2009 proporcionan información completa para análisis espaciales y temporales de la diversidad biológica.

Las mariposas son un grupo ideal para evaluar la biodiversidad por sus requerimientos ecológicos, sus respuestas a la perturbación del hábitat y a los cambios ambientales (New, 1991; Tyler et al., 1994), y por las relaciones estrechas que tanto la larva como el adulto mantienen con las plantas de alimentación. Además ofrecen un material adecuado para el análisis de patrones biogeográficos, en especial de la región neotropical (Whitmore y Prance, 1987).

La belleza de las mariposas ha impactado a las diversas culturas de todos los tiempos; están representadas en objetos de arte, se les ha empleado como símbolo, y forman parte de sus mitologías y religiones (Smart, 1975; De la Maza, 1987; New, 1991). El interés por el estudio de las mariposas mexicanas se remonta al México prehispánico (Smart, 1975; Trabulse, 1983; Beutelspacher, 1989; Tyler et al., 1994; Michán y Llorente, 2002). Sin embargo, fue hasta fines de la dominación española cuando diversos naturalistas europeos recolectaron ejemplares y se comenzaron a describir 
las especies de mariposas mexicanas. Gran parte de los ejemplares recolectados se enviaron a museos europeos. La inestabilidad del México independiente no permitió el desarrollo de la ciencia, y sólo poco a poco fue como se logró la institucionalización y con ello el establecimiento de colecciones nacionales que se fortalecieron a finales del siglo pasado. El objetivo de este artículo es integrar el conocimiento de las papiliónidas y piéridas de México a un panorama histórico de la ciencia nacional tomada de la información biológica contenida en una base de datos curatorial de papiliónidas y piéridas mexicanas.

Entre los lepidópteros, las papiliónidas y las piéridas destacan como los grupos mejor estudiados (Llorente et al., 1996), por lo que la historia de su conocimiento refleja el de las mariposas diurnas del país. El empleo de bases de datos curatoriales que aquí se aplica para reconstruir la historia del conocimiento faunístico y taxonómico de los grupos de Lepidoptera Rhopalocera de México, puede servir de modelo para estudios en otros grupos biológicos.

\section{Materiales y métodos}

La información proviene de 3 conjuntos: de la bibliografía, de ejemplares de la colección del Museo de Zoología de la Facultad de Ciencias de la Universidad Nacional Autónoma de México, y de otras colecciones de México y Estados Unidos (Llorente et al., 1997, Luis et al., 2003, 2005).

Se utilizó una base de datos en Access 2.0 con 40 752 registros que corresponden a 54559 individuos de papiliónidas y piéridas recolectados en 2341 localidades del país desde fines del siglo XIX hasta el año 2000, que se encuentran albergados en 12 museos de Estados Unidos y México, y 13807 registros o individuos documentados en 145 fuentes bibliográficas.

Los registros de cada una de las especies se ordenaron por fecha y se contaron las localidades registradas por periodos de 20 años. Las localidades se desplegaron en ArcView 3.0 y se generaron mapas de los intervalos de forma acumulativa. Se listaron y contaron las localidades visitadas por cada recolector y se obtuvieron los mapas de las localidades visitadas por los recolectores con mayor número de individuos recolectados. Se consultaron las publicaciones sobre la distribución de papiliónidas y piéridas de México (en Literatura citada con 2 asteriscos [**]) y se revisaron referencias históricas sobre papiliónidas y piéridas mexicanas, particularmente las relacionadas con la descripción de géneros y especies (en Literatura citada con el signo $\bullet$ ).

Los datos de las colecciones se presentan con su acrónimo: Allyn Museum of Entomology (Sarasota,
Florida $)=$ AME; American Museum of Natural History (Nueva York)=AMNH; California Academy of Sciences (San Francisco, California) $=$ CAS; Colección Lamberto González Cota (México, D.F.) = CLGC; Carnegie Museum of Natural History (Pittsburgh, Pennsylvania) = CMNH; Escuela Nacional de Ciencias Biológicas (México, D. F.) = ENCB; Instituto de Biología, UNAM (México, D. F.) = IBUNAM; Los Angeles County Museum (California) $=$ LACM; Museo de Zoología de la Facultad de Ciencias (Universidad Nacional Autónoma de México, México, D.F.) = MZFC; San Diego Natural History Museum (California) = SDNHM; Colección 'Essig' del Departamento de Ciencias Entomológicas, Universidad de California, Berkeley (California) = UCB; National Museum of Natural History Smithsonian Institution (Washington, D.C.) = USNM.

Los nombres de las especies y estatus taxonómico siguen la publicación de Llorente et al. (1997) sobre estas familias. El orden cronológico dado por la fecha en las etiquetas de los ejemplares es fundamental para estos resultados.

\section{Resultados}

Llorente et al. (1997) registraron 181 subespecies de Papilionidae y Pieridae integradas en 129 especies de 50 géneros pertenecientes a 5 subfamilias. Entre los autores de las descripciones de las papiliónidas y piéridas mexicanas destacan los especialistas extranjeros que cuentan con 150 taxones descritos de un total de 174, lo que representa el $86 \%$ (Fig. 1).

Las obras más destacadas del siglo XVIII son las de Cramer (1775-1782), Fabricius (1775, 1793), Herbst (1790-1804), Linnaeus (1758, 1763, 1764 y 1771) y Stoll (1786,1787 y 1791), contienen descripciones de varias especies con distribución en México. Una publicación importante de esa época fue la revista The Aurelian, editada por Moses Harris, que se estrenó con una "artística ilustración" de 2 recolectores persiguiendo mariposas (Smart, 1975).

Lo más relevante de este periodo son las descripciones de Linnaeus de 2 especies del género Battus, de Pterourus glaucus, y de 3 especies de piéridas mexicanas, así como las de Fabricius, alumno de Linnaeus, de muchas especies de insectos del mundo, reconociendo 1 147, entre las que estaban 2 papiliónidas y 2 piéridas que habitan en México. Algunos de los ejemplares que Fabricius usó para hacer las descripciones se encuentran en la colección de sir Joseph Banks en el Natural History Museum de Londres (Smart, 1975; Llorente et al., 1997). De ese tiempo no se tienen registros completos que señalen localidades precisas de los ejemplares albergados. El registro más antiguo de la base 


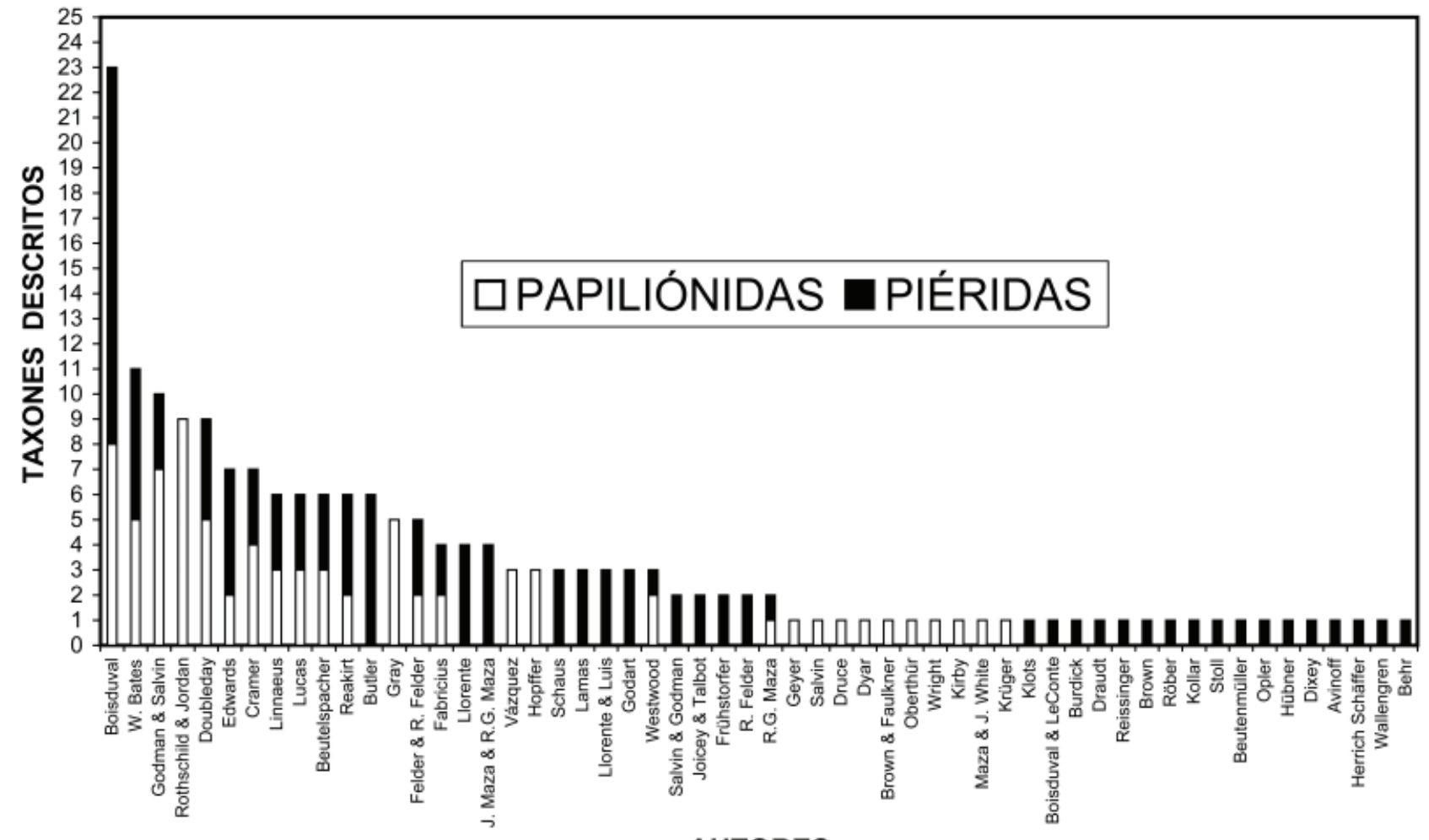

AUTORES

Figura 1. Taxones de papiliónidas y piéridas mexicanas descritas por autor.

de datos es de 1840 y se conserva en el Carnegie Museum of Natural History en Pittsburgh. Este registro corresponde a Mimoides phaon phaon, recolectada por el explorador francés Morelet en Campeche.

Durante la primera mitad del siglo XIX se publicaron las obras Suites à Buffon, Histoire naturelle des insectes de Boisduval (1836), Genera of diurnal Lepidoptera de Doubleday (1846), y Sammlung de Herrich-Schäffer (18501858). Hasta 1854 se habían descrito para México 24 taxones de papiliónidas y 27 de piéridas por importantes naturalistas, como Hübner, Geyer, Godart, Westwood, Boisduval, Doubleday, Lucas, Herrich-Schäffer y Gray.

En 1861 W. C. Hewitson publicó la obra Exotic butterflies, en 5 volúmenes con 60 láminas, a partir de ejemplares recolectados por Bates y Wallace desde 1851, donde se describió una papiliónida mexicana.

En la segunda mitad del siglo XIX destacaron las descripciones de Bates, Hopffer, Reakirt, C. Felder, R. Felder, Edwards, Butler y Oberthür, las cuales suman 14 taxones de papiliónidas y 27 de piéridas. Entre las obras más importantes de ese tiempo destacaron Reise der Osterreichischen Fregatte Novara um die Erde (1865) de Cajetan y Rudolf Felder; Lepidoptera exotica (1873-1874) de Butler, donde se incluyó la monografía del género de piéridas Callidryas (Phoebis); Synonymic catalogue of diurnal Lepidoptera, de 1871, donde W. F. Kirby reconoció 7695 especies de mariposas, contrastando con las 1147 que había reconocido Fabricius. Kirby publicó $A$ handbook to the order Lepidoptera para el Allen's Naturalist's Library (1896) y similarmente contribuyó con el manual Lloyd's Natural History (1896-1897). Casi al mismo tiempo aparecíó en Alemania Exotische Tagfalter (1884) de Staudinger, y posteriormente Exotische Schmetterlinge (1908-1909) de Röber, como contribuciones importantes a la sistemática de las mariposas, incluyendo las mexicanas. En el siglo XIX se describieron 46 taxones de papiliónidas y 61 de piéridas.

De fines del siglo XIX (1896-1899), entre las recolecciones más importantes destacan las de W. Schaus, quien recolectó ejemplares de 20 especies de papiliónidas y 27 de piéridas en el bosque mesófilo y bosque tropical subperennifolio de Veracruz (Misantla, Cuesta de Misantla, Espinal, Las Vigas, Jalapa, Veracruz, Orizaba, Huatusco, Córdoba, Zongolica y Coatzacoalcos); Nuevo León (Monterrey), Guadalajara (Jalisco); Oaxaca y San Luis Potosí. Los ejemplares se encuentran en los museos CMNH y USNM, siendo éstos los ejemplares más antiguos registrados en la base de datos.

En el CMNH, sin nombre de recolector, con fecha 
de año1899, se registraron ejemplares de Catasticta flisa y Anteos clorinde recolectados en el bosque mesófilo de Jalapa y Texolo en Veracruz, y Parides photinus, Pyrrhosticta garamas, Phoebis sennae, Eurema mexicana, Nathalis iole y Catasticta nimbice en Uruapan, Michoacán. En ese mismo año, Townsend recolectó en Chihuahua, municipio de Piedras Verdes, 3 especies de papiliónidas y 6 de piéridas de amplia distribución. En total, fueron 608 ejemplares los que se registraron en el siglo XIX.

En 1901 se concluyó Biologia Centrali Americana (volúmenes de Rhopalocera) de F. Godman y O. Salvin, en la que se cita más del $60 \%$ de las especies de papiliónidas y piéridas conocidas para México (Llorente et al., 1997; Michán y Llorente, 2002). Juntos, Godman y Salvin describieron 7 taxones de papiliónidas y 5 de piéridas; además, Salvin describió un taxón de cada familia. En esta obra monumental se registraron, entre muchos otros grupos, 34 especies de papiliónidas y 48 de piéridas obtenidas por varios recolectores. Entre los registros más numerosos destacan las especies de piéridas de amplia distribución de los géneros Eurema y Pyrisitia, las especies Zerene cesonia y Abaeis nicippe, y las papiliónidas Battus philenor y Heraclides thoas autocles. La mayor parte de estos ejemplares fueron recolectados en bosque mesófilo de Durango (Ventanas), Veracruz (Córdoba, Atoyac, Orizaba, Misantla, Coatepec, Presidio y Jalapa), Guerrero (Acapulco, Rincón, Acahuizotla, La Venta, Tepetlapa, Dos Arroyos, río Papagayo, Omiltemi), Tabasco (Teapa) y Valladolid (Yucatán).

Para el periodo entre 1900 y 1905, en los museos de CMNH, LACM, CAS, AME, AMNH y USNM se obtuvieron registros de 8 especies de papiliónidas y 20 de piéridas de amplia distribución en los estados de Baja California, Sonora, Chihuahua, Nuevo León, Tamaulipas, Sinaloa, Veracruz, Morelos y Chiapas. Entre los recolectores figuran extranjeros como Williams, Calvert, Koebele, Tower, Bishop, Conrad, Joyce, Townsend, y mexicanos como Müller y Hoffmann, de origen alemán.

En 1909, Röber describió la subespecie Colias philodice guatemalena. En los primeros años del siglo XX, Walter y Charles Rothschild contrataron recolectores para incrementar sus colecciones privadas de mariposas de todo el mundo, incluyendo las mexicanas. Entre sus recolectores se encontraba A. S. Meek, quien descubrió la especie de mariposa más grande del mundo (Smart, 1975). Parte de los resultados de estas recolecciones para América fueron publicados en la revisión de papiliónidas americanas, junto con Karl Jordan, donde se describieron 9 taxones de papiliónidas mexicanas (Rothschild y Jordan, 1906; Llorente et al., 1997). A principios del siglo XX, A. Hall recolectó en Córdoba, Huatusco, Orizaba, Motzorongo, Oaxaca, y parte de los estados de Michoacán y Morelos.
Butler y Gadow trabajaron en Jalisco, Michoacán, Morelos y Guerrero; W. Rothschild y K. Jordan los incluyeron en su revisión de papilios americanos (Rothschild y Jordan, 1906), donde registraron 26 especies de papiliónidas de Veracruz recolectadas en su mayoría por Hall, Schaus y Bilimek. En este periodo se registraron por primera vez las papiliónidas Parides sesostris zestos y $P$. panares, ambas depositadas en la colección USNM y recolectadas por Schaus.

A principios del siglo XX Roberto Müller inició la primera colección científica nacional de mariposas con ejemplares recolectados en el centro y sureste de México; con sede en la Hacienda El Mirador, Veracruz, Müller contó con el apoyo de muchos recolectores y corresponsales, como los miembros de la familia Del Toro: Rafael, Juan y Cayetano, así como A. Busck, P. Preston Clarck, M. Draudt, H. G. Dyar, G. Gugelman, M. Hering, H. Kruger, C. A. Purpus; W. Schaus, A. Seitz y L. Walsingham (Beutelspacher y Howe, 1984; De la Maza, 1987). Entre éstos, Dyar (1907) describió Battus ingenuus, y Draudt (1931) una subespecie de Pereute. En 1905, Wright describió la subespecie Papilio polyxenes coloro y, en 1907, Frühstorfer 2 especies de piéridas. La colección Müller fue una de las mayores, que quizá está dispersa en museos de Europa (Llorente y Luis, 1993; Michán et al., 2004).

En el periodo de 1906 a 1910 se registraron al menos 34 especies de papiliónidas y 28 de piéridas, de los estados de Coahuila, Tamaulipas, Sinaloa, Hidalgo, Jalisco, Michoacán, Edo. de México, Puebla, Morelos, Guerrero, Yucatán y 19 localidades de Veracruz, entre las que destacan el bosque mesófilo de Jalapa, Coatepec y Córdoba, así como la selva alta perennifolia próxima a Zongolica. Destacaron recolectores como Hoffmann, Gugelman, Del Toro, Tower, Antipovich, Barret, Calvert, Bishop, Freeman, Hopp, Müller y Schaus.

Para el periodo de 1911 a 1920, en colecciones de diversas partes del mundo se registraron 39 papiliónidas y 56 piéridas. Se aloja en CAS Anthocharis sara, en AMNH Paramidea limonea y se registra Prestonia clarki para Mazatlán, Sinaloa (Vázquez, 1956). La mayoría de estas recolecciones se hicieron en Córdoba, Veracruz, aunque también se efectuaron algunas en Sinaloa, Guanajuato, Colima, Michoacán, Estado de México, Distrito Federal, Puebla, Yucatán, Morelos y Guerrero. La mayor parte de estos ejemplares se encuentran en LACM, AMNH, CMNH, USNM y CAS. Destacan como recolectores Avinoff, Comstock, Conrad, Chopner, Gugelmann, Heid, C. Hoffmann, Kusche, Lehmann, Müller, Owen y Schaus. Una vez más, las especies más recolectadas fueron piéridas de distribución amplia de los géneros Eurema, Pyrisitia, Anteos, Zerene y Leptophobia, y especies de papiliónidas de distribución amplia, como $H$. thoas 
autocles y Pyrrhosticta victorinus. También se encontraron papiliónidas de distribución restringida, como P. photinus, Mimoides ilus y Priamides anchisiades. Boullet y Le Cerf (1912) registraron ejemplares de los géneros Parides, Battus, Protographium, Mimoides, Pyrrhosticta y Papilio resguardados en el Museo Nacional de Historia Natural de París, recolectados entre 1840 y 1911 por Boucard, Diguet, Gineste, Morelet, Owen y Schaus, en los estados de Nayarit, Veracruz, Jalisco, Puebla y Campeche.

Para la siguiente década (1921-1930) se registraron 60 especies de papiliónidas y piéridas de 69 taxones de Baja California, Veracruz y Distrito Federal como los estados mejor recolectados, y Córdoba como la localidad mejor representada. Como recolectores importantes destacan Antipovitch, quien recolectó en Veracruz, el Distrito Federal y Morelos, y Craig, con recolecciones en la península de Baja California y Sonora. En este lapso se dieron nuevos registros a 3 especies de papiliónidas $(B$. ingenuus, Pterourus palamedes y Priamides erostratus) y a 3 de piéridas (Ganyra howarthi, Zerene euridice y Pieris rapae). La mayoría de estos ejemplares se encuentran en CMNH, CAS y LACM.

Seitz en 1924 citó 11 especies de papiliónidas encontradas en Nuevo León, Veracruz, Jalisco, Colima, Michoacán, Yucatán, Morelos, Guerrero y Oaxaca en su obra Die gross Schmetterlinge der Erde. Más tarde, Joicey y Talbot (1928) describieron 2 subespecies de piéridas. Para esa década se acumularon 43 registros de especies de papiliónidas y 59 de piéridas.

En la década de 1931 a 1940 se reconocieron 31 especies de papiliónidas y 51 de piéridas con 36 y 59 taxones subespecíficos, localizados en el $60 \%$ de los estados de la República; las 10 entidades sin registros son también de las menos conocidas. Para entonces ya sumaban 70 las especies de piéridas y 50 las de papiliónidas para México. Se registraron por primera vez después de sus descripciones, las papiliónidas Papilio zelicaon, Pterourus eurymedon, Battus eracon, Pterourus rutulus y Protographium dioxippus, y las piéridas Anthocharis cethura, Aphrissa boisduvalii, Euchloe hyantis, Paramidea lanceolata, Colias alexandra, Pontia sisymbrii, Catasticta ochracea, Dismorphia crisia, Ganyra phaloe, Perrhybris pamela y Pseudopieris nehemia. Destacaron los recolectores Avinoff, Bohart, Davies, Del Toro, Escalante, Garth, Gibson, Harbison, Hoffmann, Hubbell, Klots, Martin, Michelbacher, Rindge y Smith. Resaltan las recolecciones en el bosque mesófilo de Veracruz, en las localidades de Córdoba, Jalapa y Presidio, esta última, de acuerdo con la información de la base de datos consultada, resultó ser la localidad más rica en especies de papiliónidas y piéridas de México. Tarcisio Escalante fue un importante recolector y promotor de recolecciones de ese tiempo.
En el periodo de 1929 a 1940 se inició de manera formal el estudio de las mariposas mexicanas por reconocidos zoólogos mexicanos. Fue muy importante la labor del entomólogo Carlos C. Hoffmann, quien laboraba en el recién fundado Instituto de Biología de la UNAM, donde publicó Lepidópteros nuevos de México (1). IV, Lepidópteros nuevos de México. $V$, así como el Catálogo sistemático y zoogeográfico de los lepidópteros mexicanos. Primera parte. Papilionoidea y Hesperioidea (Hoffmann, 1940a, b y c; Llorente et al., 2006), obras en las que registró 48 especies de papiliónidas y 55 de piéridas mexicanas, principalmente del bosque mesófilo y bosque tropical perennifolio de Hidalgo, Veracruz, Guerrero y Chiapas. Leonila Vázquez, alumna del maestro Hoffmann, inició la colección de lepidópteros en el Instituto de Biología de la UNAM. Poco después, la colección personal de Hoffmann fue adquirida por el American Museum of Natural History de Nueva York. En 1947, L. Vázquez describió P. erostratus erostratinus de Puebla; en 1956, la subespecie de papiliónida M. ilus occiduus de Guerrero, y en 1957, la subespecie B. philenor insularis (Vázquez, 1947, 1956 y 1957). El catálogo de Hoffmann llegó a ser la principal referencia sobre el conocimiento sistemático y distribucional de los Papilionoidea por más de 50 años, y a la fecha aún es una obra de consulta obligada para quien se inicia en el estudio de los lepidópteros de México.

En la década de 1941 a 1950 se registraron 30 especies de papiliónidas y 47 especies de piéridas, con 39 y 55 taxones subespecíficos. Como especies con nuevo registro solamente se encuentra $C$. philodice. El estado mejor representado es Veracruz, en particular el bosque mesófilo y la selva alta perennifolia de Catemaco, Fortín de Las Flores, Presidio, Tierra Blanca, Jalapa, Orizaba y Yanga. Además, están bien representados Chihuahua, Baja California Sur, Tamaulipas, San Luis Potosí y Chiapas. Destacan como recolectores de este período: Baker, Bohard, Brower, Brown, Caldwell, Davies, Dawson, Dyke, Edwards, Escalante, Harbison, Hoffmann, Hoppner, Hovanitz, Lindsley, Michener, Moore, Rindge, Ross, Stallings y Todd. En 1948, Rindge publicó un trabajo intitulado Contributions toward a knowledge of the insect fauna of Lower California, No. 8. Lepidoptera: Rhopalocera, en el que registró 2 especies de distribución amplia de papiliónidas y 12 de piéridas; además, Pontia beckeri y G. howarti para 6 localidades de Baja California y 10 de Baja California Sur. Aunque los datos de este escrito representan menos de $10 \%$ de los contenidos en la base de datos para dichos estados, es un documento preliminar sobre la especie $G$. howarti, de distribución disyunta.

De 1951 a1960 no se obtuvieron nuevas papiliónidas para México, pero en 1955 se registró por vez primera la piérida Eurema agave, descrita como la subespecie $E$. agave 
millerorum por Llorente y Luis en 1987, y se citaron en total 34 especies con 39 taxones subespecíficos de papiliónidas y 55 taxones subespecíficos de 54 especies de piéridas. Las recolecciones de Eduardo Welling son notorias con 2047 individuos en Chichen Itzá de 4 especies de papiliónidas y 21 de piéridas. Para tener más individuos recolectados, los recolectores abarcaron 24 estados, destacando Baja California Sur, Veracruz, Jalisco, Yucatán y Chiapas.

Entre los recolectores de esa década están Allen, Comstock, Chemsak, Davies, Dawson, Escalante, Giuliani, Harbison, Howe, Janzen, Malkin, Opler, Patterson, Powell, Schlinger, Spencer, Templeton y, por supuesto, Welling. La mayor parte de los ejemplares se encuentran en el CMNH, CAS, UCB, LACM, SDNHM, AMNH, $\mathrm{y}$ unos cuantos en USNM, AME, MZFC, IBUNAM y AME. Resaltan de nuevo el bosque mesófilo y la selva alta perennifolia como los tipos de vegetación preferidos.

Héctor Pérez Ruiz $(1977,1986)$ participó en el estudio de la lepidopterología en México, concentrándose en la biología de Baronia brevicornis, la especie endémica de México con el mayor número de rasgos plesiomórficos de las Papilionoidea. Para esta década ya se contaban 72 especies de piéridas.

Entre 1961 y 1970 se obtuvieron ejemplares de 38 especies de papiliónidas y 62 de piéridas, con 50 y 70 taxones subespecíficos respectivamente. Se cuenta con registros en todos los estados, siendo los mejor representados Sonora, Tamaulipas, Baja California Sur, Sinaloa, Veracruz, Morelos, Oaxaca y Chiapas. Los recolectores más destacados fueron Clench y los Miller, quienes recolectaron en Tamaulipas, Hidalgo y Veracruz principalmente; Escalante en Tamaulipas, Veracruz, Jalisco, Morelos, Guerrero y Chiapas; Howe recolectó en Tamaulipas, San Luis Potosí, Querétaro, Veracruz, y Oaxaca; R. De la Maza en Veracruz, Morelos, Oaxaca y Chiapas; Hubbellen Sonora, Chihuahua, Sinaloa,Zacatecas, Michoacán, Guerrero y Oaxaca; Jorge Llorente en el Estado de México, Distrito Federal, Tlaxcala y Morelos; Spencer en Sonora, San Luis Potosí, Veracruz, Morelos, y Oaxaca; Powell junto con Opler, en la Península de Baja California, Chihuahua, Sinaloa y Durango, y finalmente, Eduardo Welling, quien además de las copiosas recolecciones en Yucatán (Chichén Itzá), también recolectó en Zacatecas, Estado de México, Campeche, Quintana Roo, Tabasco, Oaxaca y Chiapas. La década finalizó con 74 especies de piéridas y 52 de papiliónidas reconocidas. Gary Ross citó 20 especies de papiliónidas y 37 de piéridas para la región de Los Tuxtlas de por lo menos 885 ejemplares recolectados entre 1962 y 1965 en el bosque mesófilo del cerro Vigía, Santa Marta, y la selva alta perennifolia de Sontecomapan, Catemaco, Meyacapan, San Fernando y Soteapan. Estos ejemplares están registrados en su tesis doctoral (Ross,
1967) y en publicaciones del Journal of Research on the Lepidoptera (1964-1967). En esta misma década, entre 1961 y 1970, Toliver, Holland y Cary citaron 4 especies de papiliónidas y 16 de piéridas para 6 localidades de Sonora y 21 localidades de Chihuahua (Toliver et al., 1994). En 1968 Clench publicó Butterflies from Coahuila, México, donde citó 1 especie de papiliónida y 9 de piéridas para 2 localidades de Coahuila. La mayoría de los ejemplares de esta época se encuentran alojados en CMNH, LACM, SDNHM, AMNH, CAS, UCB, MZFC, USNM y AME.

Aunque se carece de información precisa en la base de datos, se sabe que en esta década se originaron 2 de las colecciones mexicanas particulares más importantes: la de Alberto Díaz Francés y la de la familia De la Maza; la primera está alojada hoy, en el Instituto de Biología, UNAM. Algunos de los datos de estas colecciones aparecen en la base como especies registradas pero no conocemos el número de ejemplares ni las fechas precisas de recolección.

La década de 1970 tuvo gran importancia, pues se fundaron instituciones de investigación y docencia que repercutieron en el incremento de las colecciones, el aumento de especialistas y la formación de estudiantes, divulgando el conocimiento y protegiendo especies de lepidópteros (Michán et al., 2004).

En el periodo de 1971 a 1980, tiempo en el que ya se reconocían 52 especies de papiliónidas y 78 de piéridas para México, fueron registradas 49 especies de papiliónidas y 68 de piéridas de 65 y 202 taxones subespecíficos respectivamente, notablemente y bajo un gran esfuerzo de recolección. Reissinger (1972) describió la piérida Catasticta flisella. Beutelspacher (1974) describió la subespecie Troilides torquatus mazai de Jalisco, y poco después (1975a), Pterourus esperanza de La Esperanza, Oaxaca, con base en ejemplares recolectados por Javier de la Maza. Lamas (1979) describió la subespecie de Nayarit Dismorphia amphiona lupita y a P. nehemia irma. De este periodo se tienen registrados más de 12 mil ejemplares de casi todos los estados, especialmente Baja California Sur, Veracruz, Puebla, Oaxaca y Chiapas. Los estados de Baja California, Tamaulipas, San Luis Potosí, Nayarit, Colima y Morelos también están bien representados. Entre los principales recolectores destacan Lamberto González Cota, con 2733 ejemplares, y Jorge Llorente con 1 655. Además, se encuentran Howe, Olson y Spade. En esos años, Brown y Faulkner impulsaron el conocimiento de mariposas de la península de Baja California, y Tarcisio Escalante y los miembros de la familia de la Maza, por un lado, y por otro Héctor Pérez, Jorge Soberón, Enrique González y Welling recolectaron en localidades clásicas del sureste, noreste y centro del país. Carlos Beutelspacher registró 40 especies de piéridas y 43 de papiliónidas recolectadas en todos los 
estados de la República, excepto Zacatecas y Tlaxcala. En este tiempo Roberto G. de la Maza describió una subespecie de papiliónida y una piérida, y 4 subespecies de piéridas, en colaboración con Javier de la Maza.

Para la década de 1981 a 1990 se citaron 52 especies con 61 subespecies de papiliónidas y 72 especies con 86 subespecies de piéridas. Este periodo fue sumamente rico en recolecciones, descripciones y trabajos taxonómicos y faunísticos sobre lepidópteros; se cuenta con más de 6500 registros de 11560 individuos. Las piéridas C. nimbice, Lieinix nemesis y Eurema albula se encuentran entre las especies más recolectadas. Por otro lado, se citaron erróneamente especies que tal vez no estén en México, como Papilio machaon bairdii, P. indra pergamus y Parides childrenae latifasciata, y se buscó Parnassius, infructuosamente, en el noreste de México.

Lamas (1981) describió Glutophrissa drusilla tenuis. R. G. de la Maza describió la subespecie Protographium thyastes occidentalis de Guerrero (De la Maza et al., 1982). En 1984 Llorente describió 2 subespecies: Enantia mazai diazi para Nayarit y la típica E. mazai mazai para Veracruz, así como la subespecie de Nayarit L. nemesis nayaritensis y la subespecie $D$. amphiona isolda de Oaxaca. En este mismo año, J. de la Maza Elvira y R. G. de la Maza E. (1984) describieron la subespecie de Chiapas Lieinix lala turrenti y la especie L. neblina de Guerrero, así como la subespecie de Chiapas D. crisia alvarezi y la oaxaqueña Melete polyhymnia serrana. Beutelspacher describió la subespecie de Durango Eucheira socialis westwoodi, (Beutelspacher, 1984), la subespecie $P$. erostratus vazquezae de Guerrero (Beutelspacher, 1986a) y la subespecie C. flisa oaxaca y C. teutila flavifaciata (Beutelspacher, 1986b), ambas de Oaxaca. Opler (1986) describió la piérida Euchloe guaymasensis, Javier de la Maza y los hermanos White describieron la subespecie $B$. brevicornis rufodiscalis del centro de Chiapas (De la Maza et al., 1987).

Roberto de la Maza Ramírez (1987) publicó Mariposas mexicanas: guía para su colecta y determinación, donde citó 43 especies de papiliónidas y 52 de piéridas para 229 localidades de 25 estados, entre los que destacan localidades de bosque mesófilo y selva alta perennifolia de Veracruz, Puebla, Oaxaca y Chiapas; y selva baja caducifolia de Morelos y Guerrero. Esta obra representa una compilación de información sumamente importante. La mayor parte de los ejemplares de este periodo se alojan en MZFC, LACM, SDNHM, CAS, UCB, USNM, CLGC, AMNH, CMNH, AME, BMNH e INIA.

En 1988 Llorente y Luis describieron las subespecies Dismorphia eunoe popoluca y D. eunoe chamula para Veracruz y Chiapas, respectivamente. J. de la Maza y R. G de la Maza (1989) describieron 2 subespecies $P$. pamela chajulensis para el este de Chiapas y P. pamela mapa para el oeste.

Los estados de Baja California Sur, San Luis Potosí, Veracruz, Distrito Federal, Puebla, Morelos, Guerrero, Oaxaca y Chiapas cuentan con más del $80 \%$ de los individuos registrados para ese periodo de tiempo. Las localidades de Teocelo y Jalapa en Veracruz, barranca de Patla en Puebla, Atoyac de Álvarez en Guerrero, y Chiltepec en Oaxaca, fueron las mejor conocidas. Entre los recolectores más importantes del periodo están Jorge Llorente y Armando Luis; el último realizó su tesis de licenciatura en el bosque mesófilo y el bosque de Abies de Los Dínamos, en el Distrito Federal; figuran también Isabel Vargas, quien recolectó en San Rafael, Vicente Aranda en el derrame del Chichinautzin, Morelos, Yolanda Bizuet en el Parque Nacional El Chico, Hidalgo, y Alma Garcés y Lamberto González Cota en varias localidades de bosque mesófilo del país; Roberto G. de la Maza, Roberto R. de la Maza, Javier de la Maza, y A. Díaz Francés recolectaron en localidades del sureste de México y algunos estados de la costa del Pacífico.

Beutelspacher y Howe (1984) citaron 52 especies de papiliónidas en el primer fascículo de Mariposas de México. Fascículo I: Introducción y generalidades, superfamilia Papilionoidea; familia Papilionidae. Entre las especies raras que mencionaron están: P. childrenae latifasciata, Protographium marcellus marcellus, Papilio indra pergamus y P. machaon bairdii, de dudosa representación en México.

Llorente y Luis (1987) describieron E. agave millerorum de Tapachula y Teapa en el estado de Tabasco, y los de la Maza (1989) a M. polyhymnia florinda para la selva lacandona (Chiapas) y Metates (Oaxaca). Brown y Faulkner(1982) recolectaron más de 600 ejemplares en Baja California, Sonora, Baja California Sur y Sinaloa, citando 5 especies de papiliónidas y 25 de piéridas. La mayoría de los ejemplares de esta década se alojan principalmente en el Museo de Zoología de la Facultad de Ciencias de la UNAM, y en orden decreciente en SDNHM, LACM, UCB, CLGC, CMNH, USNM, CAS, DGSV, IBUNAM, AMNH y ENCB.

Como resultado del trabajo de este periodo en la siguiente década, Luis y Llorente (1990), Luis et al. (1991) y Vargas et al. (1991) citan más de 65 especies de papiliónidas y piéridas de la sierra de Atoyac y Omiltemi en Guerrero, la sierra de Juárez en Oaxaca, y del Valle de México. También apareció un libro importante de Tyler et al. (1994) sobre papiliónidas de América. Finaliza esta década contabilizando 52 especies de papiliónidas y 78 de piéridas, sin registros de especies desconocidas para papiliónidas o piéridas mexicanas (Llorente et al., 1997; Lamas, 2004 y Llorente et al., 2006). 
Brown y Faulkner (1992) describieron la subespecie Calaides astyalus bajaensis de Baja California Sur. Finalmente, Llorente et al. (1997) editaron el libro Papilionidae y Pieridae de México: distribución geográfica e ilustración, que compila los registros conocidos de las papiliónidas y piéridas de México en las principales colecciones institucionales, así como la bibliografía correspondiente. En este libro se grafica la distribución de papiliónidas y piéridas de México, se reconocen 129 especies de 50 géneros y 5 subfamilias, y se presentan
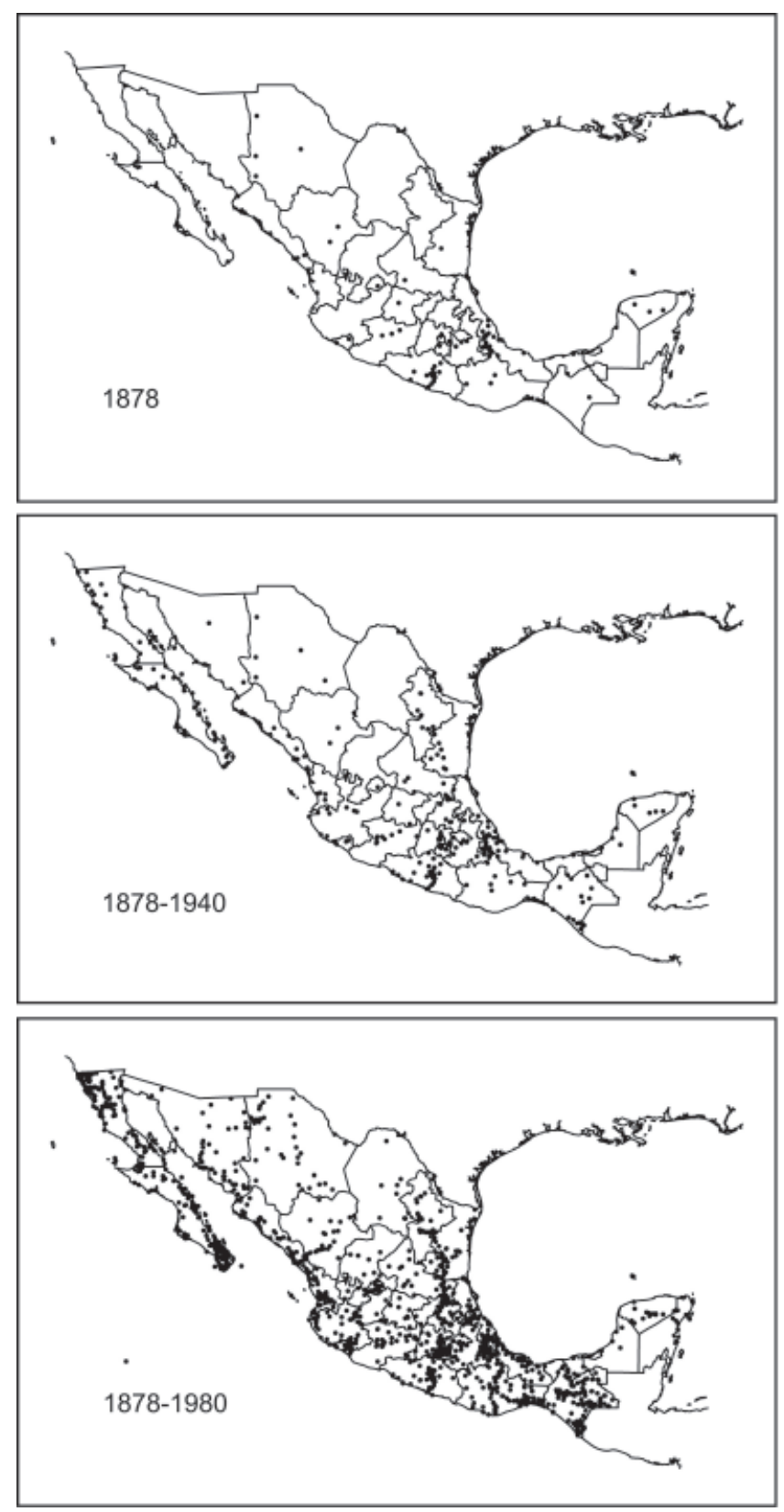

los mapas de las localidades de registro y 29 láminas con las especies de las 2 familias. Todo esto al acopiar más de 5 decenas de miles de ejemplares en la base de datos MARIPOSA (Luis et al., 2001, 2003, 2006).

En la figura 2 se muestran las localidades de recolección acumuladas por cada periodo y en la figura 3, las localidades por periodo. Del total de mariposas recolectadas para México, destacan como los más visitados los siguientes estados: Baja California Sur con 244 localidades, Veracruz con 238, Baja California con 203, Chiapas con 174, Oaxaca
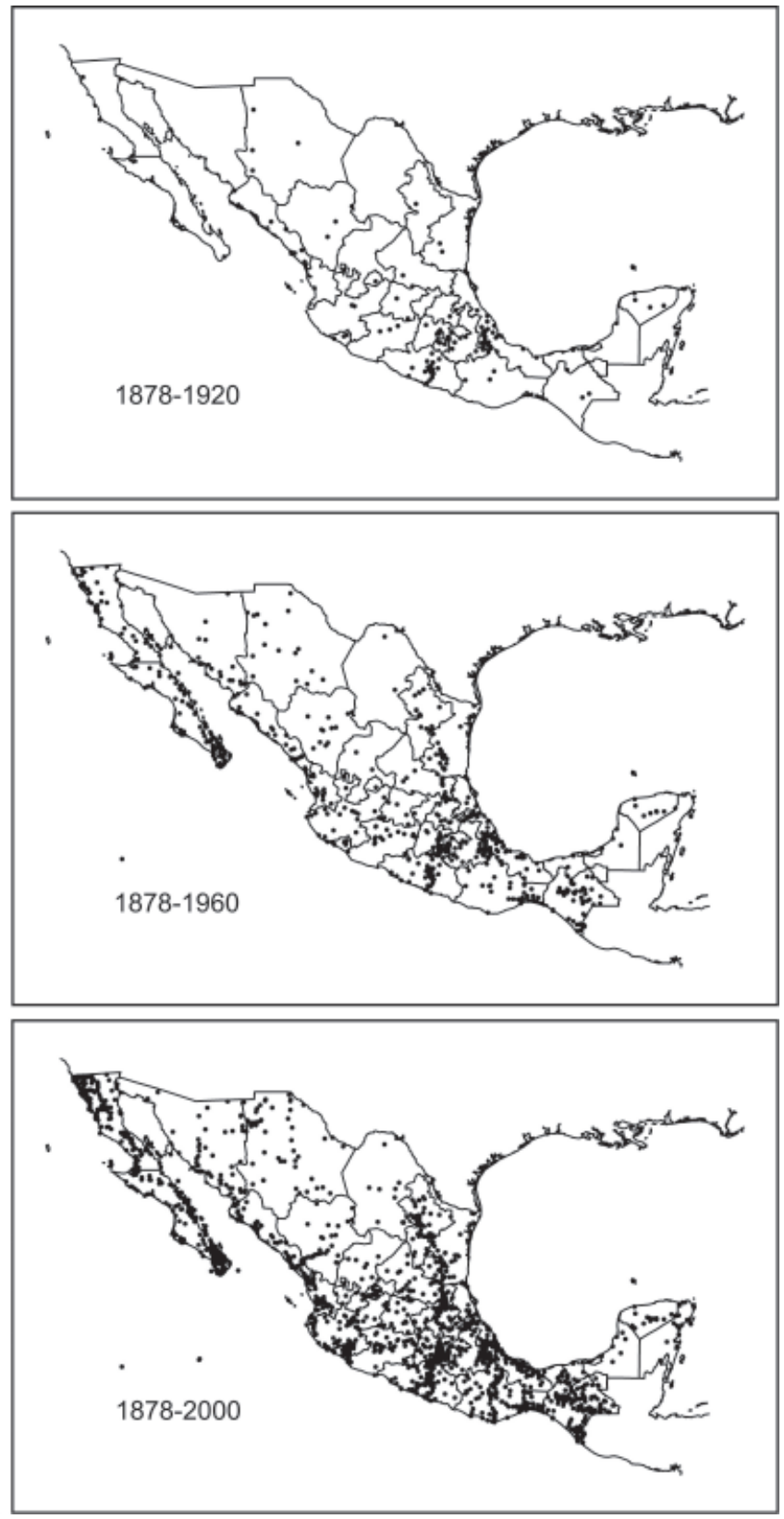

Figura 2. Localidades acumulativas donde se han recolectado papiliónidas y piéridas por periodo de 2 décadas. 
con 153 y Guerrero con 132. La parte norte del país refleja un esfuerzo de recolecta menor (Oñate et al., 2000). Los 2 estados de la península de Baja California tienen un alto número de localidades y recolecciones, con un número bajo de especies, mientras que los estados del sur han sido los más explorados y los que tienen mayor número de especies de papiliónidas y piéridas. Las localidades y los puertos de Veracruz y Acapulco influyeron con mayor frecuencia entre los distintos recolectores, seguramente porque fueron los puntos de llegada a México de las embarcaciones
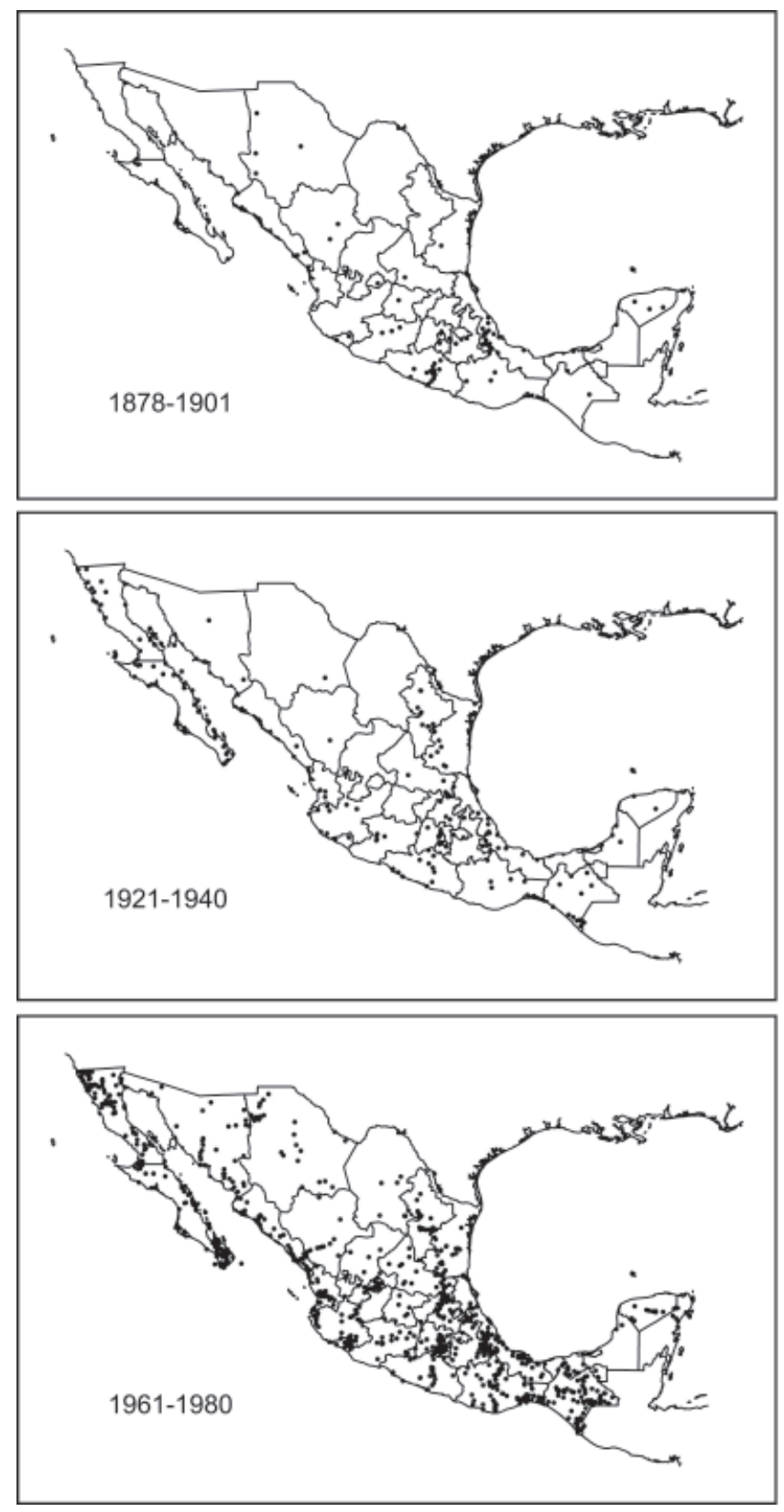

europeas hasta principios del siglo XX. La mayoría de las localidades de estos 2 estados siguen el efecto carretera o síndrome de las vías de acceso (Soberón et al., 2000; Espadas et al., 2003). En menor grado se conocen los estados de Tamaulipas, San Luis Potosí, Aguascalientes, Guanajuato y Tlaxcala (Llorente et al., 1993).

En Veracruz las recolecciones recurrentes se concentraron en la región de Los Tuxtlas y en el bosque mesófilo de montaña de la zona de Orizaba, Córdoba, Jalapa Teocelo, Texolo y Fortín de las Flores; por tanto,
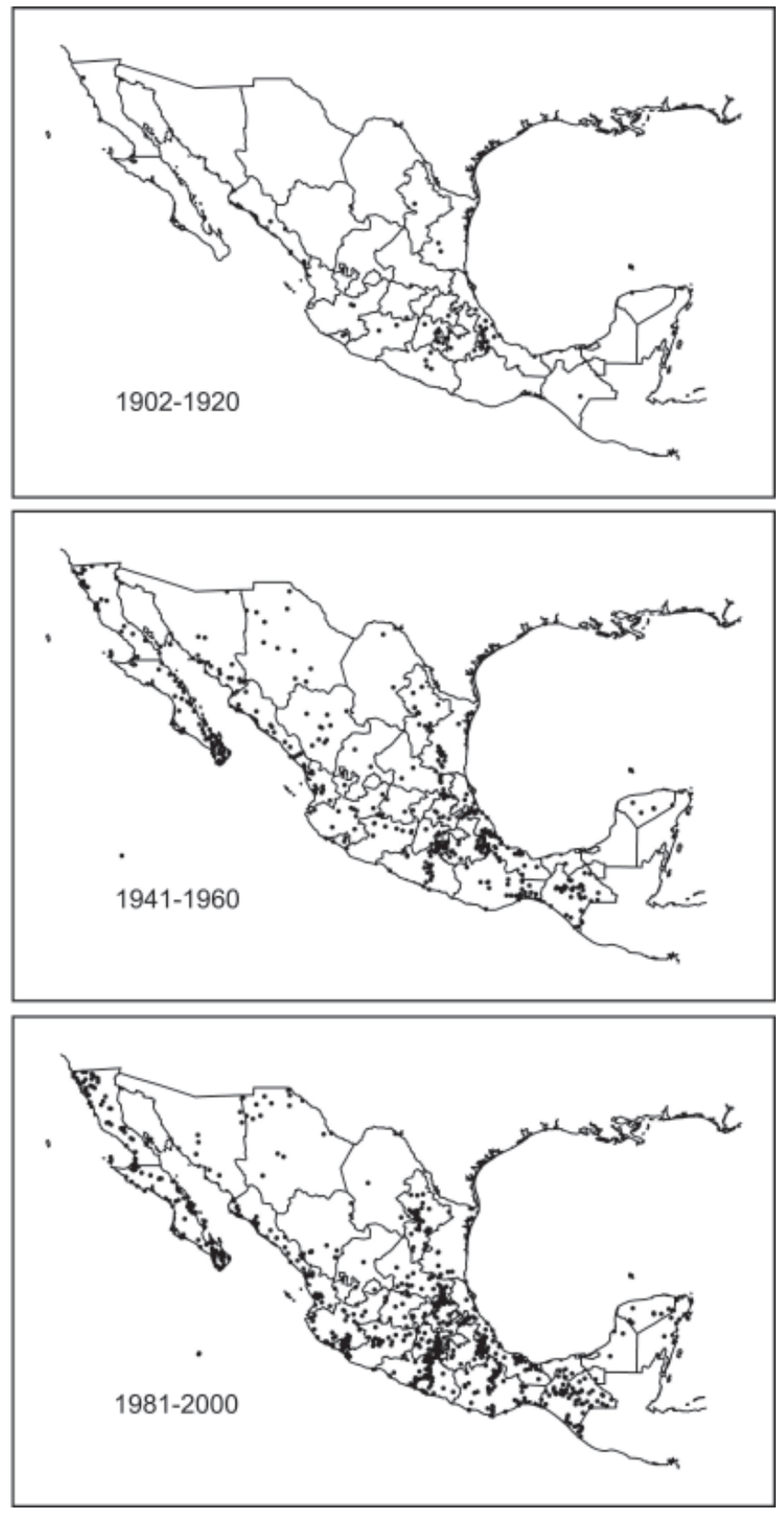

Figura 3. Localidades donde se han recolectado papiliónidas y piéridas por periodo de 2 décadas. 
son estas pocas localidades las que se conocen mejor; se localizan entre los 18 y $19^{\circ} \mathrm{N}$ y 95 y $97^{\circ} \mathrm{O}$. En Puebla hay una concentración alta de localidades para papiliónidas y piéridas en la zona de Patla, Tequesquitla y La Ceiba, entre los 20 y $21^{\circ} \mathrm{N}$ y 97 y $98^{\circ} \mathrm{O}$; el resto de las localidades de Puebla tienen registros aislados y poca intensidad de recolecta. En Chiapas los registros se concentran en Chajul, Comitán, Mapastepec, Tacaná y Palenque. La mayor incidencia de recolecciones se ubica entre los $16 \mathrm{y}$ $17^{\circ} \mathrm{N}$ y 92 y $94^{\circ} \mathrm{O} ; 15$ y $16^{\circ} \mathrm{N}$ y 92 y $93^{\circ} \mathrm{O}$ y 16 y $17^{\circ}$ $\mathrm{N}$ y 90 y $91^{\circ} \mathrm{O}$. En Oaxaca los sitios mejor recolectados se encuentran en varias localidades de la sierra de Juárez, Loxicha (Candelaria), Chiltepec, Jacatepec, La Esperanza, La Soledad, Metates, Puerto Eligio y Valle Nacional, que se encuentran entre 17 y $18^{\circ} \mathrm{N}$ y 96 y $97^{\circ} \mathrm{O}$ y entre 15 y $16^{\circ} \mathrm{N}$ y 96 y $97^{\circ} \mathrm{O}$, respectivamente. La mayoría de las localidades de Oaxaca son las asociadas a las carreteras y son registros aislados. En Guerrero se observan puntos de baja intensidad de recolecta por toda la carretera desde Acapulco hacia el Distrito Federal; por ejemplo, Chilpancingo, Iguala y Taxco; sin embargo, las recolecciones bien establecidas son las de Omiltemi, Atoyac de Álvarez, arroyo Las Damas, cañón del Zopilote, Ixcateopan de Cuauhtémoc, Mezcala y Acahuizotla. Las localidades más visitadas se concentran entre 17 y $18^{\circ} \mathrm{N}$ y 99 y $101^{\circ} \mathrm{O}$, y entre 18 y $19^{\circ}$ $\mathrm{N}$ y 99 y $100^{\circ} \mathrm{O}$. En Morelos reinciden las recolecciones en Cuernavaca, Rancho Viejo, cañón de Lobos, Cuautla, el derrame del Chichinautzin, Tepoztlán y Yautepec. En Morelos los sitios mejor conocidos se encuentran entre los 18 y $19^{\circ} \mathrm{N}$ y 99 y $100^{\circ} \mathrm{O}$.

En la figura 4 se presentan las localidades visitadas por los recolectores con más registros en la base de datos: Carlos Beutelspacher, Lamberto González Cota, Jorge Llorente y Armando Luis, y los miembros de la familia De la Maza. Algunos lugares han sido visitados recurrentemente por los distintos recolectores en distintas épocas, convirtiéndose en localidades clásicas por la riqueza de especies que contienen,como es el caso de diversos puntos del centro y sureste mexicano con bosque mesófilo de montaña y bosque tropical perennifolio. A manera de resumen se preparó el Cuadro 1 que también sirve para la Discusión.

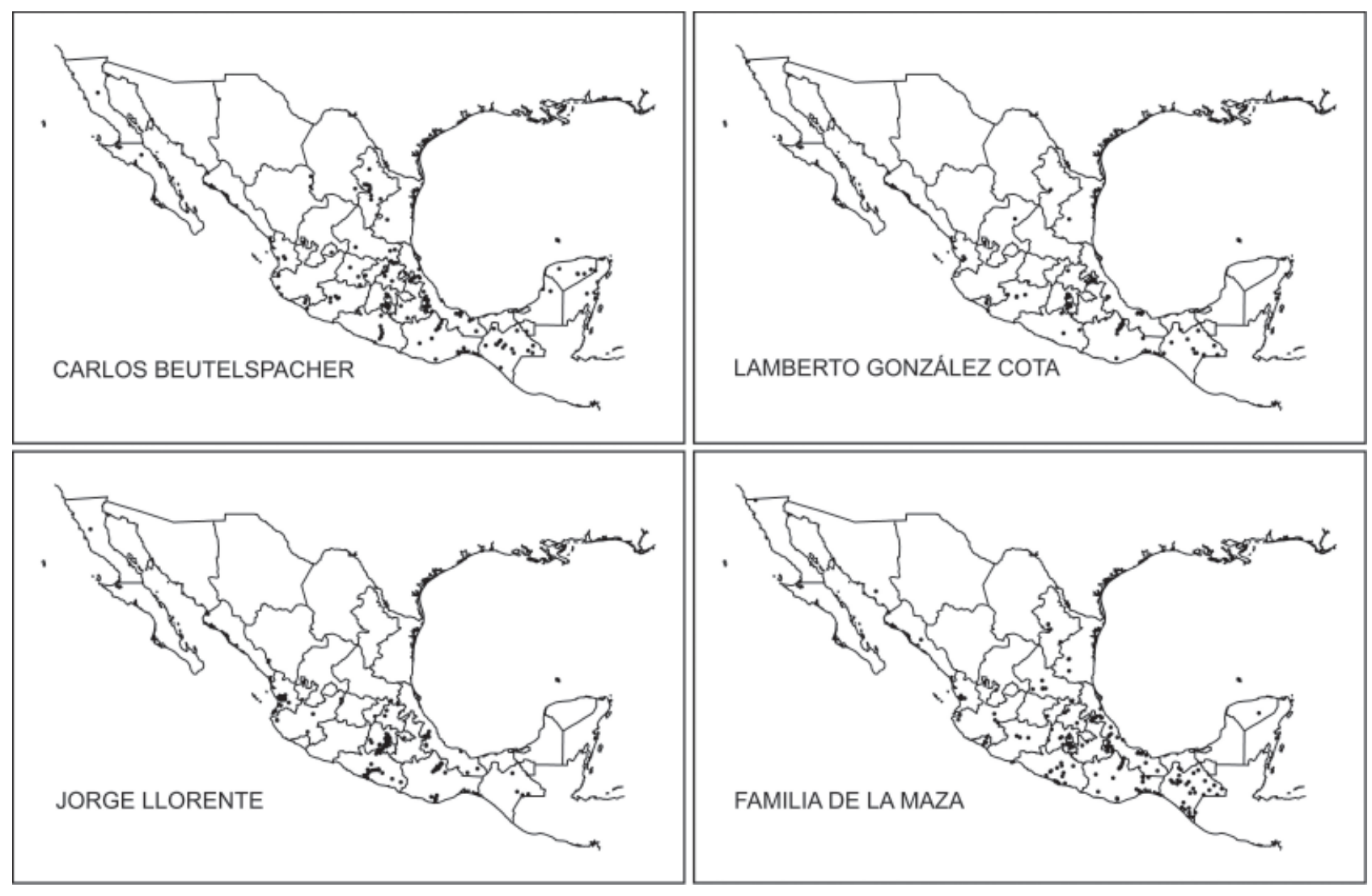

Figura 4. Localidades visitadas por los colectores más importantes. 
Cuadro 1. Resumen por periodos de descripciones de taxones, individuos y taxones registrados de papiliónidas y piéridas mexicanas

\begin{tabular}{|c|c|c|c|c|c|c|c|c|c|}
\hline \multirow{2}{*}{ Periodo } & \multicolumn{2}{|c|}{ Descripciones } & \multirow{2}{*}{$\begin{array}{c}\text { Descrip. } \\
\text { totales }\end{array}$} & \multicolumn{2}{|c|}{ Individuos registrados } & \multirow{2}{*}{$\begin{array}{c}\text { Individuos } \\
\text { totales }\end{array}$} & \multicolumn{2}{|c|}{ Taxones registrados } & \multirow{2}{*}{$\begin{array}{c}\text { Taxones } \\
\text { totales }\end{array}$} \\
\hline & Papiliónidas & Piéridas & & Papiliónidas & Piéridas & & Papiliónidas & Piéridas & \\
\hline S-XVIII & 8 & 8 & 16 & 0 & 0 & 0 & 0 & 0 & 0 \\
\hline S-XIX & 46 & 61 & 107 & 92 & 516 & 608 & 13 & 16 & 29 \\
\hline $1901-1910$ & 10 & 3 & 13 & 170 & 353 & 523 & 34 & 48 & 82 \\
\hline $1911-1920$ & 0 & 3 & 3 & 82 & 167 & 249 & 39 & 56 & 95 \\
\hline 1921-1930 & 0 & 3 & 3 & 61 & 501 & 562 & 22 & 47 & 69 \\
\hline $1931-1940$ & 0 & 2 & 2 & 289 & 1251 & 1540 & 48 & 59 & 107 \\
\hline 1941-1950 & 1 & 0 & 1 & 346 & 910 & 1256 & 39 & 55 & 94 \\
\hline $1951-1960$ & 1 & 0 & 1 & 407 & 3521 & 3928 & 44 & 64 & 108 \\
\hline $1961-1970$ & 0 & 0 & 0 & 760 & 3023 & 3783 & 50 & 70 & 120 \\
\hline 1971-1980 & 2 & 3 & 5 & 3382 & 9560 & 12942 & 65 & 202 & 156 \\
\hline $1981-1990$ & 3 & 19 & 22 & 2452 & 9108 & 11560 & 61 & 86 & 147 \\
\hline \multirow[t]{2}{*}{ 1991-1995 } & 1 & 0 & 1 & 11 & 37 & 48 & 7 & 18 & 25 \\
\hline & & & 174 & & & 36999 & & & \\
\hline
\end{tabular}

\section{Discusión}

De los 54559 individuos de papiliónidas y piéridas con registro en la base, sólo 36999 cuentan con datos completos para el análisis de la información que se resume en el Cuadro 1. Mientras que en el siglo XVIII se describieron 16 papiliónidas y piéridas, pero no se cuenta con ejemplares, durante el siglo XIX se describió un gran número de papiliónidas (46) y piéridas (61) que coincide con un aumento de la literatura, representada por las publicaciones de Boisduval, Doubleday, Herrich-Schâffer, Bates, C. Felder, que contrasta con el bajo número de ejemplares (608) de esa época, Del siglo XIX sólo hay registro de 29 taxones.

En la primera década del siglo XX fueron muy importantes las contribuciones de Godman y Salvin (1878-1901), Rothschild y Jordan (1906) y los esfuerzos de Müller por coleccionar mariposas mexicanas, pues el número de taxones registrados se elevó a 82 , a pesar que los ejemplares registrados fueron menos que en el siglo XIX, de tan sólo 523 ejemplares. En la primera década del siglo XX solamente se llevaron a cabo 13 descripciones de papiliónidas y piéridas. El número de taxones registrados se incrementó ligeramente en la década de 1911 a 1920, lo que coincidió con la obra de Seitz (1906-1932), la más importante del siglo XX sobre mariposas del mundo (Smart, 1975; Llorente et al., 1997). En México el desarrollo de la lepidopterología fue frenado por la inestabilidad política del país durante la Revolución (Michán y Llorente, 2002). A pesar de los esfuerzos, los avances en la formalización de la lepidopterología se perdieron, disolviéndose las sociedades, revistas e instituciones que ya se habían creado, y gran cantidad de ejemplares y colecciones de ese periodo se enviaron a museos de diversas partes del mundo (Michán et al., 2004).

En la década de 1921 a 1930 ya se conocían para el país 43 especies de papiliónidas y 59 de piéridas, a pesar del bajo número de ejemplares registrados (562). Fue hasta la fundación del Instituto de Biología de la Universidad Nacional Autónoma de México a fines de 1929, y de la Escuela Nacional de Ciencias Biológicas del Instituto Politécnico Nacional en 1933, que se impulsó el estudio de las mariposas y muchas otras áreas de la biología (Michán y Llorente, 2002, 2003; Michán et al., 2004). Al crearse los Anales del Instituto de Biología, se publicaron los trabajos de Carlos Hoffmann, quien desde 1932 produjo información importante de estas 2 familias y que culminó con la publicación del Catálogo sistemático y zoogeográfico de los lepidópteros de México en 1940-1942.

De este modo, durante la década de 1931 a 1940 se triplicó el número de individuos registrados, aunque es notorio que los ejemplares fueron depositados en AMNH, LACM y SDNHM, a pesar que muchos fueron recolectados por Carlos Hoffmann. Los taxones registrados ascendieron en esta década a 107, reconociéndose el $92 \%$ de las papiliónidas mexicanas y el $70 \%$ de piéridas. En la década de 1941 a 1950 se mantuvo el número de ejemplares registrados así como los taxones conocidos. Lo mismo ocurrió durante la década de 1951 a 1960. En la década de 1961 a 1970 volvió a incrementarse el número de taxones 
registrados, aunque en los trabajos de Leonila Vázquez, Carlos R. Beutelspacher y Héctor Pérez no se describieron nuevos.

En las décadas de 1971 a 1980 y 1981 a 1990 se incrementaron significativamente los ejemplares recolectados, lo que se refleja también en un aumento de taxones registrados para el país. Durante esta década se iniciaron organizaciones de investigación y docencia relevantes, como el Instituto de Ecología, A.C. (1974) y el Museo de Zoología de la Facultad de Ciencias de la UNAM (1978). Además, fueron financiados proyectos de investigación y se formó la Sociedad Mexicana de Lepidopterología, con el objetivo de incrementar las colecciones, divulgar el conocimiento y proteger especies importantes de lepidópteros (Michán et al., 2004).

Con la finalidad de impulsar y fortalecer el desarrollo de la ciencia, en 1970 se creó el Consejo Nacional de Ciencia y Tecnología (CONACYT) que desde entonces apoya en sus actividades a estudiantes, investigadores e instituciones en general (Michán y Llorente, 2002) y los estudios lepidopterológicos en particular.

En la década de los años ochenta (1984) se inició el Sistema Nacional de Investigadores (SNI) para el fomento, estímulo, eficiencia y calidad de la investigación en México. Sin embargo, en casi 25 años de existencia, el SNI ha beneficiado a no más de 60 entomólogos (Michán y Llorente, 2002), entre los cuales han estado 8 lepidopterólogos.

Entre 1981 y 1990 se describieron 19 taxones de piéridas y 3 de papiliónidas, lo que representó un avance significativo en el conocimiento de papiliónidas y piéridas mexicanos. En los últimos años se incrementó la compilación de datos sobre estas 2 familias y otros lepidópteros, debido a la apertura de proyectos financiados por la Comisión para el Conocimiento y Uso de la Biodiversidad (CONABIO), lo que impulsó el desarrollo de bases de datos e investigación en zonas de gran riqueza biótica o poco explorados (CONABIO, 1997). Han sido varios los proyectos del Museo de Zoología de la Facultad de Ciencias financiados por la CONABIO y que han fomentado, entre otras investigaciones, la que se concluye en este artículo, en conjunto con dependencias que apoyan la investigación en la Universidad Nacional Autónoma de México.

La mayor parte de las recolecciones han incidido en bosque tropical perennifolio, bosque tropical caducifolio y bosque mesófilo de montaña. Entre los recolectores, Luis Lamberto González Cota aportó gran número de ejemplares y realizó una labor relevante en el conocimiento de sitios de interés, por lo que su colección fue adquirida por el Museo de Zoología de la Facultad de Ciencias en 1995 (Michán et al., 2004). Por otro lado, la colección de la familia De la Maza ha sido de gran importancia para el conocimiento de las mariposas mexicanas. Los De la Maza recolectaron principalmente en bosque mesófilo y selva alta perennifolia de los sitios más concurridos de Veracruz, Puebla, Oaxaca y Chiapas, y en la selva baja caducifolia de Morelos y Guerrero. Además de los sitios clásicos, inauguraron áreas de recolección con alto número de endemismos y gran riqueza de Papilionoidea.

Cabe destacar que 33\% de los ejemplares registrados en la base de datos se alojan en el Museo de Zoología, donde se han realizado investigaciones detalladas en Guerrero, Jalisco, Colima, Cuenca del Valle de México, Veracruz y Oaxaca (Luis y Llorente, 1990; Luis et al., 1991; Vargas et al., 1991, 1994; Luis et al., 1995; Vargas et al., 1996; Warren et al., 1996, 1998; Luis et al., 1999; Vargas et al., 1999; Luis et al., 2004), además de recolecciones en Sinaloa, Durango y Chiapas. El 95\% del total de los registros corresponden a recolecciones del siglo $\mathrm{XX}$, mientras que sólo se ha recuperado el 5\% de la información anterior. Por otro lado, sólo el $8 \%$ de las papiliónidas y y el $15 \%$ de las piéridas fueron descritas por mexicanos, entre los que destacan Roberto y Javier de la Maza, Leonila Vázquez, Carlos Beutelspacher, Jorge Llorente y Armando Luis. Apoya estos resultados lo descrito por otros autores, quienes señalan que la mayoría de los datos de ejemplares recolectados en las regiones de alta biodiversidad no se encuentran en colecciones nacionales, sino en museos de otras partes del mundo (Barrera, 1974; Michán y Llorente, 2002; Navarro et al., 2003b; Michán et al., 2004).

De este modo podemos concluir que el conocimiento de papiliónidas y piéridas en colecciones se perfeccionó en las últimas 3 décadas del siglo XX; posiblemente la mayor parte de los ejemplares recolectados en la primera mitad del siglo XX y en siglos anteriores, se han perdido o se encuentran dispersos en colecciones extranjeras, sin sistematizar. Para mejorar el conocimiento de las papiliónidas y piéridas mexicanas es necesario completar la base de datos con los ejemplares que se encuentran dispersos en diversas colecciones europeas, por lo que es preciso apoyar proyectos de investigación que promuevan la adquisición de esta información. Del mismo modo, es preciso trabajar con las autoridades de los distintos museos para que preparen o sistematicen en bases de datos los ejemplares guardados durante tanto tiempo y que podrían proporcionar información valiosa acerca de las regiones exploradas, las rutas que tomaron los naturalistas y la distribución de la diversidad de mariposas mexicanas recolectadas en esa época. Dada la posibilidad de que algunas especies sean indicadoras de hábitats poco perturbados, el conocimiento de los datos geográficos en etiquetas de ejemplares recolectados durante el siglo XIX sea de valor para estudios de reconstrucción de las áreas de 
distribución originales.

El análisis de las bases de datos curatoriales permite detallar y enriquecer la descripción e interpretación de las cronologías del conocimiento faunístico; la faunística es una base factual importante de la zoología. Al sistematizar o graficar los datos curatoriales de ejemplares se pueden resaltar los periodos históricos y áreas geográficas de incremento de muestreo de las poblaciones. El número de ejemplares por si solo puede ser evidencia, respecto al tiempo, del aumento en el conocimiento de las faunas y de las áreas de distribución de las especies que comprende; sin embargo, la asociación temporal del esfuerzo de recolección en faunística sólo es una variable, ya que si la asociación se efectúa con los colectores, las publicaciones, la creación de instituciones académicas y de apoyo a la ciencia, o bien las regiones geográficas de modo distinto clasificadas, los resultados son mucho mayores e interesantes para los análisis del progreso científico de las disciplinas taxonómicas. En este trabajo se ha mostrado que la fundación del Instituto de Biología (1929), la investigación en la Facultad de Ciencias (1974), ambas de la Universidad Nacional Autónoma de México, así como la creación de la Sociedad Mexicana de Lepidopterología son hitos en la historia de esta disciplina y en el incremento del muestreo de este taxón en diversas regiones de México, pues promovieron los estudios faunísticos y su registro es discernible en las colecciones formadas y las bases de datos curatoriales que se han formado de ellas.

Las bases de datos curatoriales en conjunto con bases de literatura científica y bases de datos taxonómicas o meras listas sistemáticas de un taxón, conforman una tríada que permite análisis más profundos del desarrollo histórico en una disciplina taxonómica. Tales análisis son indispensables para planificar el curso futuro y énfasis de muestreo de un taxón en un territorio dado, como en este trabajo se ha mostrado en relación con otros de los mismos autores (Oñate et al., 2000; Soberón et al., 2000, Michán et al., 2004).

\section{Agradecimientos}

A Cristina Cramer Hemkes, Juan José Morrone Lupi, Jorge Meave del Castillo, Adolfo Navarro Sigüenza, Layla Michán, Graciela Zamudio y Pedro Miramontes, por la lectura y sugerencias al manuscrito final, así como a Armando Luis Martínez, Isabel Vargas Fernández, Aquiles Bernal, Minerva García, Jimena Castro y Marisol Trujano, por su colaboración. Los proyectos 212006 y 203509 de DGAPA y 83237 de CONACyT fueron de gran apoyo para el desarrollo de este trabajo.

\section{Literatura citada}

(** relacionada con la distribución de papiliónidas y piéridas;

- relacionada con descripción de género o especie) .

**Abud, Q. G. 1987. Aspectos ecológico y taxonómico de insectos (orden Lepidoptera e Hymenoptera) en el Bosque-Escuela de la sierra de la Primavera. Tesis, Universidad de Guadalajara. Guadalajara, Jalisco. 323 p.

**Abud, Q. G. 1988 Aspectos ecológico y taxonómico de insectos (orden Lepidoptera e Hymenoptera) en el Bosque-Escuela de la sierra de la Primavera. Amatl. Boletín del Instituto de Madera, Celulosa y Papel de la Universidad de Guadalajara 2:12-21.

**Acuña, A. L. 1990. Mariposas diurnas (Lepidoptera: Papilionoidea y Hesperoidea) del Rancho El Jaguiey, Gabriel Zamora, Michoacán. Tesis, Universidad Michoacana de San Nicolás de Hidalgo. Morelia, Michoacán. 97 p.

**Adams, J. K. 1983. An old first United States record finally published: Papilio victorinus (Papilionidae) in Laredo, Texas. Journal of Lepidopterist's Society 37:13-18.

**Balcázar, M. A. 1988. Fauna de Mariposas de Pedernales, Municipio de Tacámbaro, Michoacán (Lepidoptera: Papilionoidea y Hesperoidea). Tesis, Universidad Michoacana de San Nicolás de Hidalgo. Morelia, Michoacán. 89 p.

Barrera, A. 1974. Las colecciones científicas y su problemática en un país subdesarrollado: México. Biología 4:12-19

**Barrera, A. y M. E. Díaz-Batres. 1977. Distribución de algunos lepidópteros de la sierra de Nanchititla, México, con especial referencia a Tisiphone maculata Hpff. (Insecta:Lepidoptera) Revista de la Sociedad Mexicana de Lepidopterología 3: 17-28.

**Barrera, T. G y L. H. Romero. 1986. Estudio faunístico de lepidópteros (superfamilia Papilionoidea) en un bosque mesófilo de montaña en Cascada de los Diamantes, San Rafael, Estado de México. Tesis, ENEP Zaragoza, Universidad Nacional Autónoma de México, México, D.F. $58 \mathrm{p}$.

**Behr, H. H. 1890. Lepidoptera from San José del Cabo. Zoe 50:246-247.

**Beutelspacher B., C. 1974. Reconsideración taxonómica de Papilio tolus G. y S. (Lepidoptera: Papilionidae) y descripción de una nueva subespecie. Revista de la Sociedad Mexicana de Historia Natural 35:149-157.

**Beutelspacher B., C. 1975a. Una especie nueva de Papilio L. (Papilionidae). Revista de la Sociedad Mexicana de Lepidopterología 1:3-6.

**Beutelspacher B., C. 1975b. Notas sobre el suborden Rhopalocera (Lepidoptera) de Las Minas, Veracruz. Revista de la Sociedad Mexicana de Lepidopterología 1:11-20.

**Beutelspacher B., C. 1976. Nuevas formas de papiliónidos mexicanos. Revista de la Sociedad Mexicana de Lepidopterología 2:61-70.

**Beutelspacher B., C. 1981 Lepidópteros de Chamela, Jalisco, México. I. Rhopalocera. Anales del Instituto de Biología, Universidad Nacional Autónoma de México, Serie Zoología 52:371-388.

**Beutelspacher B., C. 1982. La familia Pieridae (Lepidoptera) 
en el estado de Nuevo León, México. Anales del Instituto de Biología, Universidad Nacional Autónoma de México, Serie Zoología 53:367-378.

**Beutelspacher B., C. 1982b. Mariposas diurnas de "El Chorreadero", Chiapas (Insecta: Lepidoptera). Anales del Instituto de Biología, Universidad Nacional Autónoma de México, Serie Zoología 53:341-366.

**Beutelspacher B., C. 1984. Una nueva subespecie de Eucheira socialis Westwood (Lepidoptera: Pieridae) de México. Anales del Instituto de Biología, Universidad Nacional Autónoma de México, Serie Zoología 54:111-118.

**Beutelspacher B., C. 1986a. Una nueva subespecie mexicana de Papilio erostratus Westwood (Insecta, Lepidoptera, Papilionidae). Anales del Instituto de Biología, Universidad Nacional Autónoma de México, Serie Zoología 56:241-244.

**Beutelspacher, B., C. 1986b. Algunas observaciones taxonómicas sobre el género Catasticta Butler en México, con la descripción de una nueva subespecie (Lepidoptera: Pieride). Anales del Instituto de Biología, Universidad Nacional Autónoma de México, Serie Zoología 57:153-160.

**Beutelspacher, B., C. 1986c. Adiciones a los piéridos mexicanos (Lepidoptera: Pieridae). Anales del Instituto de Biología, Universidad Nacional Autónoma de México, Serie Zoología 56:647-649.

Beutelspacher, B., C. 1989. Las mariposas entre los antiguos mexicanos. Fondo de Cultura Económica, México, D.F. $104 \mathrm{p}$.

**Beutelspacher B., C. y H. Brailovsky, 1979. Notas sobre depredación de lepidópteros por hemípteros. Anales del Instituto de Biología, Universidad Nacional Autónoma de México, Serie Zoología 50:777.

**Beutelspacher B., C. y M. G. Beutelspacher. 1976. Ciclo de vida de Falcapica limonea Butler (Lepidoptera: Pieridae). Revista de la Sociedad Mexicana de Lepidopterología 2: 32-34.

**Beutelspacher, C y W. Howe. 1984. Mariposas de México. Fascículo 1. Prensa Médica Mexicana, México, D.F. 128 p. + 20 láms.

-Boisduval, J. B. A. D. 1836. Suites à Buffon. Histoire naturelle des insectes. Spécies general des lépidoptéres. Librairie Encyclopédique Roret, Paris. 1:4+xii+690+6p., pls.1A-4A, 1B-12B, 1C-8c.

**Boullet, E. y F. L. Le Cerf. 1912. Catalogue de la collection de Lépidoptéres de Muséum National D'Histoire Naturelle de Paris. I. Famille Papilionidae. Imprimérie Nationale, Paris. iv $+47 \mathrm{p}$.

**Brower, L. P. 1958. Speciation in butterflies of the Papilio glaucus Group I. Morphological relationship and hybridization. Evolution 13:40-63.

**Brown, F. M. 1943. Notes on mexican butterflies I. Papilionidae. Journal of New York Entomological Society 5:161-178.

**Brown, F. M. 1944. Notes on mexican butterflies II. Pieridae. Journal of New York Entomological Society 5:99-119.

**Brown, J. W. 1990. Additions to the Papilionoidea(Lepidoptera) of the Revillagigedo Islands, Mexico. Entomological News 101:167-169.

**Brown, J. W. y D. K. Faulkner. 1982. New Rhopalocera records for Baja California with the description of a new species of
Habrodais Scudder (Lepidoptera:Theclinae). Bulletin of Allyn Museum 67:1-6.

**Brown, J. W. y D. K. Faulkner. 1984. Distributional records of certain Rhopalocera in Baja California, Mexico, with the description of a new subspecies of Papilio (Heraclides) astyalus (Godart) (Lepidoptera: Papilionidae) Bulletin of Allyn Museum 83:1-9.

**Brown, J. W. y D. K. Faulkner. 1988. The butterflies of isla de Cedros, Baja California Norte, México. Journal of Research on the Lepidoptera 27:233-256.

Butler, A. G. 1870. A revision of the genera of the subfamily Pierinae. Cistula Entomologica 1:33-58

-Butler, A. G. 1873-1874. Lepidoptera Exotica, or descriptions and illustrations of exotic Lepidoptera. E. W. Janson, London. p. 153-162, pls. 55-57 (1873); 163-174, pls. 58-60 (1874).

Clench, H. K. 1968. Butteflies from Coahuila, Mexico. Journal of the Lepidopterists' Society 22:227-231.

**Clench, H. K. 1971. Two new hairstreaks from México (Lepidoptera:Lycaenidae). Bulletin of Allyn Museum 3:1-6.

CONABIO (Comisión Nacional para el Conocimiento y Uso de la Biodiversidad). 1998. La diversidad biológica de México. Comisión Nacional para el Conocimiento y Uso de la Biodiversidad, México, D.F. 267 p.

**Comstock, J. A. y L. Vázquez. 1960. Estudio de los ciclos biológicos en lepidópteros mexicanos. Anales del Instituto de Biología, Universidad Nacional Autónoma de México 31:349-448.

-Cramer, P. 1775-1782. De uitlandische Kapellen voorkomende in de drie waereld-Deelen Asia, Africa, en America-Papillons exotiques des trois parties du monde l'Asie, l'Afrique et l'Amerique. S. J. Baalde and J. Van Schoonhoven, Amsterdam/Utrecht. Barthelemy Wild 1(1/7): i-xxx, 1-16, 1-132, pls. 1-84; (8): 133-155, pls. 85-96 (1776); 2(9/16):1151, pls. 97-192 (1777); 3(17/22): 1-128, pls. 193-264 (1779); (23/24):129-176, pls. 265-288(1780); 4(25/28):1-90, pls. 289-336 (1780), (29/32):91-192, pls. 337-384 (1781); (33/34):193-252, pls. 385-400 (1782).

**D'Almeida, R. F. 1966. Catálogo dos Papilionidae Americanos. Sao Paulo, Sociedade Brasileira de Entomologia. 366 p.

**De Almeida, C. R. 1977. Mariposas de Tabasco. Boletín informativo de la Sociedad Mexicana de Lepidopterología 3:5-7.

**De la Maza E., J. 1975. Una nueva forma de Prestonia clarki Schaus (Pieridae). Revista de la Sociedad Mexicana de Lepidopterología 1:38-41.

**De la Maza E., J. y A. Díaz F. 1979. Notas y descripciones sobre la familia Papilionidae en México. Revista de la Sociedad Mexicana de Lepidopterología 4:51-56.

**De la Maza E., J. y A. White. 1986. Redescubrimiento de Nymphalis cyanomelas (Dbld. \& Hew.) en México. (Nymphalidae: Nymphalinae). Revista de la Sociedad Mexicana de Lepidopterología 10:35-39.

**De la Maza, E., J. y A. White. 1990. Rhopalocera de la Huasteca potosina, su distribución, composición, origen y evolución. Revista de la Sociedad Mexicana de Lepidopterología 13:31-88.

**De la Maza E., J., J. White y A. White. 1987. Observaciones 
sobre el polimorfismo femenino de Baronia brevicornis Salv. (Papilionidae:Baroniinae) con la descripción de una nueva subespecie del estado de Chiapas, México. Revista de la Sociedad Mexicana de Lepidopterología 11:3-13.

**De la Maza E., J. y R. De la Maza E. 1976. Papiliónidos del cañón del Novillo, Tamaulipas (Lepidoptera: Papilionidae). Revista de la Sociedad Mexicana de Lepidopterología 2:24-31.

**De la Maza E., J. y R. De la Maza E. 1984. Nuevos Dismorphiinae de México y El Salvador (Pieridae). Revista de la Sociedad Mexicana de Lepidopterología 9:3-12.

**De la Maza E., J. y R. De la Maza E. 1985. La fauna de Mariposas de Boca de Chajul, Chiapas, México, (Rhopalocera). Parte I. Revista de la Sociedad Mexicana de Lepidopterología 9:23-44.

**De la Maza E., J. y R. De la Maza E. 1989. Notas sobre Perrhybris pamela (Cramer), con la descripción de dos nuevas subespecies de México y Guatemala (Pieridae: Pierinae). Revista de la Sociedad Mexicana de Lepidopterología 13:3-10.

**De la Maza E., J., R. De la Maza E. y R. De la Maza R. 1982. Lepidópteros nuevos del Estado de Guerrero, México. (Papilionoidea). Revista de la Sociedad Mexicana de Lepidopterología 7:2-14.

**De la Maza E., R. 1975. Notas sobre Lepidópteros de Rancho Viejo y Tepoztlán, Morelos, México. Primera parte: Papilionoidea. Revista de la Sociedad Mexicana de Lepidopterología 1:42-61.

**De la Maza E., R. 1980. Las poblaciones centroamericanas de Parides erithalion (Boisd). (Papilionidae: Troidini). Revista de la Sociedad Mexicana de Lepidopterología 5:51-74.

**De la Maza E., R. y A. Díaz F. 1978. Una nueva subespecie de Parides lycimenes Boisd. de México (Lepidoptera, Papilionidae) Revista de la Sociedad Mexicana de Lepidopterología 4:7-14.

**De la Maza E., R. y E. Olaya 1979. Hallazgo de una población de Papilio abderus Hopf. en la sierra de Álvarez, San Luis Potosí, México (Papilionidae) Boletín Informativo de la Sociedad Mexicana de Lepidopterología 5:7-9.

**De la Maza E., R. y J. De la Maza E. 1979. Confirmación de la existencia de Parides lycimenes lycimenes Boisd. en la región Lacandona, Chiapas, México. (Papilionidae) Revista de la Sociedad Mexicana de Lepidopterología 4:47-50.

**De la Maza E., R. y J. De la Maza E. 1988. Notas sobre los Rhopalocera de la sierra de Álvarez, San Luis Potosí, México. (Lepidoptera). Revista de la Sociedad Mexicana de Lepidopterología 11:33-59.

**De la Maza E., R. y R. De la Maza R. 1978. Notas sobre los Papilionidae en México (LEP.) IV. Área de Orizaba a Yanga. Boletín Informativo de la Sociedad Mexicana de Lepidopterología 4:15-30.

**De la Maza E., R. y R. De la Maza R. 1979. Notas sobre los Papilionidae en México. V. Zona de Los Tuxtlas, Veracruz. Boletín Informativo de la Sociedad Mexicana de Lepidopterología 5:2-18.

**De la Maza R., R. 1976a. Una interesante aberración de Parides alopius (Godman y Salvin) (Papilionidae). Revista de la Sociedad Mexicana de Lepidopterología 2:5-7.
**De la Maza R., R. 1976b. Colecta en sierra de Juárez, Oaxaca. Boletín Informativo de la Sociedad Mexicana de Lepidopterología 2:2-4.

**De la Maza R., R. 1976c. Colecta del 14 al 23 de abril en los estados de Oaxaca y Chiapas. Boletín Informativo de la Sociedad Mexicana de Lepidopterología, 2:6-7.

**De la Maza R., R. 1976d. Colecta en el sureste. Boletín Informativo de la Sociedad Mexicana de Lepidopterología $1: 2-5$.

**De la Maza R., R. 1977. Nueva forma femenina de Catasticta teutila Dbld. (Pieridae). Revista de la Sociedad Mexicana de Lepidopterología 3: 33-34.

**De la Maza R., R. 1984. Descripción de una nueva subespecie de Melete florinda Butler, de Oaxaca, México. (Pieridae) Revista de la Sociedad Mexicana de Lepidopterología 9:19-20.

**De la Maza R., R. 1987. Mariposas mexicanas. Guía para su colecta y determinación. Fondo de Cultura Económica, México, D.F. 304 p.

**Del Río, A. 1978. Algunas observaciones sobre la biología, hábitos y enemigos naturales del "gusano perro": Papilio garamas garamas Hübner en la región de Uruapan, Mich. Tesis, Universidad Michoacana de San Nicolás de Hidalgo, Morelia, Michoacán. 36 p.

**Díaz F., A. 1975a. Relato de un viaje al sureste de México. Revista de la Sociedad Mexicana de Lepidopterología 1:23-24.

**Díaz F., A. 1975b. Papiliónidos del valle de Tepoztlán, Morelos. Boletín Informativo de la Sociedad Mexicana de Lepidopterología 1:5-7.

**Domínguez, Y. y J. L. Carrillo. 1976. Lista de insectos en la Colección Entomológica de Investigaciones Agrícolas. Segundo suplemento. Folleto Misceláneo 29. Instituto Nacional de Investigaciones Agrícolas, Secretaría de Agricultura y Ganadería. iv +245 p.

-Doubleday, E. 1846. The genera of diurnal Lepidoptera: comprising their generic characters, a notice of their habits and transformations, and a catalogue of the species of each genus. Longman, Brown, Green \& Longmans, London. 1: 1-6 pls., A, 1-2. 7-18 pls. 3-4.

**Doubleday, E. y J. O. Westwood. 1846-1850. The genera of diurnal Lepidoptera. Longman, Brown, Green \& Longmans, London. 1:1-41.

Espadas M. C., R. Durán y J. Argáez. 2003. Phytogeographic analysis of taxa endemic to the Yucatán Peninsula using geographic information systems, the domain heuristic method and parsimony analysis of endemicity. Diversity and Distributions 9:313-330.

-Fabricius, J. C. 1775. Systema entomologiae, sistens insectorum classes, ordines, genera, species, adiectis synonymis, locis, descriptionibus, observationibus. Korte, Flensburgi et Lipsiae, . [iv $]+[\mathrm{xii}]+[\mathrm{xvi}]+832 \mathrm{p}$.

-Fabricius, J. C. 1793. Entomologia systematica emendata et aucta. Secundum classes, ordines, genera, species adjectis synonimis, locis, observationibus, descriptionibus. Christian Gottlieb Proft., Fil. et Soc., Hafniae, 3: iv +487 p.

-Felder, C. y R. Felder. 1865. Reise der österreicheschen Fregatte Novara um die Erde in den Jahren 1857, 1858, 1859 unter 
den Befehlen des Commodore B. von Wüllerstorf. Urbair. Zoologischer Theil. Zweiter Band. Zweite Abtheilung: Lepidoptera. Carl Gerold's Sohn, Wien (1): 4+1-136 pls 121,(2): 2+137-378, pls 22-47. (3): 2+379-536 pls, 48-74. (4): $6+1-9$.

**Ferris, C. D. y J. F. Emmel. 1982. Discussion of Papilio coloro W.G. Wright (= Papilio rudkini F.\& R. Chermock) and Papilio polyxenes Fabricius (Papilionidae). Bulletin of the Allyn Museum 76:1-13

**García M., G. 1982. Estudio taxonómico ecológico de la flora y fauna del estado de Aguascalientes I. Inventario y distribución de la fauna entomológica (Lepidoptera) de Aguascalientes. Universidad Autónoma de Aguascalientes, Aguascalientes. $116 \mathrm{p}$.

**Gibson, W. y J. L. Carrillo. 1959. Lista de insectos en la Colección Entomológica de la Oficina de Estudios Especiales, S.A.G. Folleto Misceláneo 9. Secretaría de Agricultura y Ganadería, México, D.F. 254 p.

**Godman, F. D. e I. O. Salvin. 1878-1901. Biologia Centrali Americana. Insecta, Lepidoptera Rhopalocera. Dulach, London. 2:782.

**González C., L. 1977. Reporte de la Colecta en la Ceiba, Puebla. Boletín informativo de la Sociedad Mexicana de Lepidopterología 3:6-7.

**González C., L. 1978. Notas sobre la Familia Papilionidae (Lepidoptera), en México. Barranca de Patla, Pue., y alrededores. Boletín informativo de la Sociedad Mexicana de Lepidopterología 4:3-15.

**González C., L. y C. Velázquez M. 1977. Nueva forma de Catasticta flisa H-S. Revista de la Sociedad Mexicana de Lepidopterología 3:91-92.

**Guzmán, E. P. 1976. Algunas observaciones sobre Lepidópteros de Chalma, Estado de México. Revista de la Sociedad Mexicana de Lepidopterología 2:49-51.

-Herbst, J. F. W. 1790-1804. In Natursystem aller bekannten in -und ausländischen Insekten als eine Fortsetzung der von Buffonschen Naturgeschichte. Nach dem System des Ritters Carl von Linné bearbeitet. Der Schmetterlinge, C. G. Jablonsky, (ed.), Joachim Pauli, Berlin.1: [22] + cxxviii + 1216 pls. [1] + 1-6 prontsp. (1783); $2:$ xxxii + 1-295 pls. 7-20 (1784); 3:1-113 pls. 21-37 (signatures A-H) (1788).

**Hernández B. F. 1989. Mariposas diurnas del municipio de Jalapa, Veracruz. (Insecta: Lepidoptera) México. Taxonomía, ecología y zoogeografía. Tesis, Facultad de Biología. Universidad Veracruzana. $154 \mathrm{p}$.

**Hernández, V. 1985. Mariposas del suborden Rhopalocera (Lepidoptera) de Amealco, Querétaro y alrededores. Tesis, Universidad Nacional Autónoma de México. México, D.F. $99 \mathrm{p}$.

**Hernández, V., H. I. Martínez G. y S. Rodríguez N. 1981. Lepidópteros en la Colección Entomológica de la Dirección General de Sanidad Vegetal. Fitófilo 84:15-17.

Herrich-Schäffer, G, A. W. 1850-1858. Sammlung neuer oder wenig bekannter aussereuropäischer Schmetterlings. G.J. Manz, Regensburg.1:1-52(1856), 53-84 (1858), pls. [110](1850), 11-14(1853), 15-18(1855), 19-22(1856), 23-24 (1858).

•Hewitson, W. C. 1861. Illustrations of new species of exotic butterflies, selected chiefly from the collections of W. Wilson Saunders and William C. Hewitson. van Voorst. London. 2: 5-6, 39-40, 87-88 pls. 3, 20, 44, (40): 13-14, 49-50, 69-70 + I-iv + 121-124 pls. $7,25,35$.

**Hoffmann, C. C. 1932. Roberto Müeller y su importancia en el conocimiento de los lepidópteros de México. In memoriam. Anales del Instituto de Biología, Universidad Nacional Autónoma de México 3:133-148.

**Hoffmann, C. C. 1933. La fauna de lepidópteros del distrito del Soconusco (Chiapas). Un estudio zoogeográfico. Anales del Instituto de Biología, Universidad Nacional Autónoma de México 4:207-307.

**Hoffmann, C. C. 1936. Contribuciones al conocimiento de la fauna de Actopan, Hgo. I. Algunas observaciones de la fauna de lepidópteros en la época seca. Anales del Instituto de Biología, Universidad Nacional Autónoma de México 7:259-263

**Hoffmann, C. C. 1940a. Lepidópteros nuevos de México (1). IV. Anales del Instituto de Biología, Universidad Nacional Autónoma de México 11:275-284.

**Hoffmann, C. C. 1940b. Lepidópteros nuevos de México. V. Anales del Instituto de Biología, Universidad Nacional Autónoma de México 11:633-638.

**Hoffmann, C. C. 1940c. Catálogo sistemático y zoogeográfico de los lepidópteros mexicanos, primera parte. Papilionoidea. Anales del Instituto de Biología, Universidad Nacional Autónoma de México 11:730-739.

**Holland, R. 1973. Butterflies of middle and Southern Baja California. Journal of Research on the Lepidoptera 11:147-160.

•Hübner, J. 1806-1838. Zuträge zur Sammlung Exotischer Schemetterlinge, published by author and by Geyer after Hübner's death. Augsburg. 3 vols.,.named but not numbered plates with short, unpaginated text. $1: 213$ pls. 1806-1819; 36 pls. 1807-1824; 2:221 pls., 1819-1826; 4 pls., 1827 (Geyer); 6 pls., 1827-1832 (Geyer); 353 pls., 1827-1838 (Geyer); CFC, pc).

**Johnson, K. y D. Matusik. 1989. A study of Protesilaus microdamas (Burmeister) and the little-known P.dospassosi (Rutimeyer) and P. huanucana (Varea de Luque) (Papilionidae). Journal of Research on the Lepidoptera 27:83-95.

-Joicey, J. y G. Talbot. 1928. New forms of Rhopalocera in Hill Museum. Bulletin of the Hill Museum. 2:19-27

**Jurado, C. 1990. Inventario de Lepidópteros diurnos del Vivero Forestal "Lázaro Cárdenas", Municipio de Morelia, Michoacán. Tesis, Universidad Michoacana de San Nicolás de Hidalgo, Morelia, Michoacán. 46 p.

**Katthain, G. 1971. Estudio taxonómico y datos ecológicos del suborden Rhopalocera (Insecta, Lepidoptera en un área del Pedregal de San Ángel, D.F., México. Tesis, Universidad Nacional Autónoma de México, México, D.F. 189 p.

-Kirby, W. F. 1884. On a copy of Peale's Lepidoptera Americana in the library of the Zoological Department of the British Museum. Papilio 4:103-104.

Kirby, W. F. 1896. A handbook to the order Lepidoptera, part 1. Butterflies 2. In Allen's Naturalist's Library. W. H. Allen, London xvi +332 p. 68 pls. 
Kirby, W. F. 1896-1897. Lloyd's natural history: a handbook to the order Lepidoptera. London. 1478 p.158 pls.

**Kendall, R. O. y W. W. McGuire. 1984. Some new and rare records of Lepidoptera found in Texas. Bulletin of Allyn Museum 86:1-50.

**Lamas, G. 1979. Los Dismorphiinae (Pieridae) de México, América Central y las Antillas. Revista de la Sociedad Mexicana de Lepidopterología 5:3-37.

Lamas, G. 1981. Notes on Peruvian butterflies (Lepidoptera). VI. Twelve new Pieridae. Revista de Ciencias [Lima] 73:44-53.

Lamas, G. 2004. Atlas of Neotropical Lepidoptera. Checklist: part 4a. Hesperioidea-Papilionoidea. Lamas G. (Ed). Mc Guire Center for Lepidoptera and Biodiversity Association for Tropical Lepidoptera. Scientific Publishers, Gainesville, Florida. 439 p.

-Linnaeus, C. 1758. Systema naturae per regna tria naturae, secundum classes, ordines, genera, species, cum characteribus, differentiis, synomymis, locis. Editio decima, reformata. Laurentius Salvius, Holmiae. 1: iv $+823+\{1\}$ p. (Reprint in MidContinent Lepidoptera Series 2(23) April, 1971).

-Linnaeus, C. 1763. Amoenitates Academicae; seu dissertationes variae physicae,medicae, botanicae, antehac seorsim editae, nunc collectae et auctae cum tabulis aeneis. Laurentius Salvius, Holmiae. 6: iv +486 p., 5 pls.

-Linnaeus, C. 1764. Museum S:ae R:ae M:tis Ludovicae Ulricae Reginae Svecorum, Gothorum, Vandalorumque \&c. \&c. \&c. In quo animalia rariora, exotica, imprimis Insecta \& Conchilia describuntur \& determinantur. Podromi instar editium. Laurentius Salvius, Holmiae. [viii]+720 + [2] p.

-Linnaeus, C. 1771. Regni animalis, Appendix. Insecta. p. 529543. In Mantissa plantarum altera generum editionis VI \& specierum editionis II. Laurentius Salvius, Holmiae. p. [i-vi], 143-588.

**Llorente B., J. 1984. Sinopsis sistemática y biogeográfica de los Dismorphiinae de México con especial referencia al género Enantia Hübner (Lepidoptera: Pieridae). Folia Entomologica Mexicana 58:1-207

**Llorente B., J. 1986. Las razas geográficas de Pereute charops (Boisduval, 1836) con la descripción de una nueva subespecie (Lepidoptera: Pieridae). Anales del Instituto de Biología, Universidad Nacional Autónoma de México, Serie Zoología 56: 245-258.

-**Llorente B., J. y A. Luis. 1987. Una nueva subespecie de Eurema agave Cramer (Lepidoptera: Pieridae; Coliadinae). Folia Entomologica Mexicana 71: 17-25.

**Llorente B., J. y A. Luis. 1988. Nuevos Dismorphiinae de México y Guatemala (Lepidoptera: Pieridae). Folia Entomologica Mexicana 74: 159-178.

Llorente B., J. y A. Luis. 1993. Conservation-oriented analysis of mexican butterflies: Papilionidae (Lepidoptera: Papilionoidea). In Biological diversity of Mexico: origins and distribution, T. P. Ramamoorthy, R Bye, A. Lot y J. Fa (eds.), Oxford University Press, New York. p. 147-177.

Llorente B., J., A. Luis e I. Vargas. 2006. Apéndice general de Papilionoidea: Lista sistemática, distribución estatal y provincias biogeográficas. In Componentes principales de la entomofauna mexicana, Morrone, J. J. y J. Llorente
Bousquets (eds.), Las Prensas de Ciencias, Facultad de Ciencias, UNAM, México, D.F. p. 945-1009.

Llorente B., J., A. Luis, I. Vargas y J. Soberón M. 1993. Biodiversidad de las mariposas: su conocimiento y conservación en México,. Revista de la Sociedad Mexicana de Historia Natural, volumen especial 44:313-324.

Llorente B., J., I. Vargas y J. Soberón. 1996. Papilionoidea (Lepidoptera) In Biodiversidad, taxonomía y biogeografía de artrópodos de México: hacia una síntesis de su conocimiento, J. Llorente B., A. García y E. González (eds.). Instituto de Biología, UNAM, México. D. F. p. 531-548.

Llorente B., J., L. Oñate-Ocaña, A. Luis-Martínez e I. Vargas F. 1997. Papilionidae y Pieridae de México: distribución geográfica e ilustración. Comisión Nacional para el Conocimiento y Uso de la Biodiversidad / Universidad Nacional Autónoma de México, México, D.F. 229 p.

**López, G. B. A. 1989. Mariposas del suborden Rhopalocera (Lepidoptera) de la barranca de Huentitan, Guadalajara, Jalisco (registro de especies). Tesis, Universidad Autónoma de Guadalajara, Jalisco. 90 p.

**Luis, M. A. 1987. Distribución altitudinal y estacional de los Papilionoidea (Insecta:Lepidoptera) en la cañada de Los Dínamos, Magdalena Contreras, D. F. Tesis, Facultad de Ciencias,Universidad Nacional Autónoma de México, México, D.F. 113 p.

Luis, M. A. y J. Llorente B. 1990. Mariposas del Valle de México: introducción e historia. 1. Distribución local y estacional de los Papilionoidea de la cañada de Los Dinamos; Magdalena Contreras, D.F. México. Folia Entomologica Mexicana 78: 95-198

Luis, M. A., J. Llorente e I. Vargas. 2005. Una megabase de datos de mariposas de México y la regionalización biogeográficaa. In Regionalización biogeográfica en Iberoamérica y tópicos afines: primeras jornadas biogeográficas RIBES, J. Llorente y J. J. Morrone (eds.); Las Prensas de Ciencias, Facultad de Ciencias, UNAM, México. D. F. p. 269-294.

Luis, A., J. Llorente, A. D. Warren e I. Vargas. 2004. Los lepidópteros:papilionoideos yhesperioideos.In Biodiversidad de Oaxaca, A. J.García-Mendoza, M. J. Ordóñez y M. A. Briones-Salas (eds.), Instituto de Biología, UNAM / Fondo Oaxaqueño para la Conservación de la Naturaleza/ WWF, México, D. F. p. 335-355.

Luis, M. A., I. Vargas y J. Llorente. 1991. Lepidopterofauna de Oaxaca I: Distribución y fenología de los Papilionoidea de la sierra de Juárez. Publicaciones especiales del Museo de Zoología 3. Facultad de Ciencias, Universidad Nacional Autónoma de México, México. D.F. 3:1-121.

Luis, M. A., I. Vargas y J. Llorente. 1995. Síntesis de los Papilionoidea (Rhopalocera: Lepidoptera) del estado de Veracruz. Folia Entomológica Mexicana 93:91-133.

Luis, M. A., I. Vargas y J. Llorente. 1999. Mariposas de las áreas montanas del sur de la Cuenca de México. In Biodiversidad de la región de montaña del sur de la Cuenca de México. Velázquez, A. y F. Romero (eds.), Universidad Autónoma Metropolitana,. México, D.F. p. 188-203.

Luis, M. A., I. Vargas y A. D. Warren. 2003. Biodiversity and biogeography of Mexican butterflies (Lepidoptera: Papilionoidea and Hesperioidea. Proceedings of the 
Entomological Society of Washington 105: 209-224.

Martín-Piera, F. y J. Lobo. 2003. Database records as a samplingeffort surrogate to predict spatial distribution of insects in either poorly or unevenly surveyed areas. Acta Entomológica Ibérica e Macaronésica 1:23-35.

**Mathieu, J. M. y C. A. González. 1970. Lista de insectos en la Colección Entomológica del Instituto Tecnológico de Monterrey. Ocho familias de Papilionoidea (Lepidóptera). Boletín de Agronomía, Escuela de Agricultura y Ganadería, ITESM 129:10-15.

**Mattoni, R. H. T. 1988. The Euphilotes battoides complex: recognition of a species and description of a new subespecies (Lycaenidae) Journal of Research on the Lepidoptera 27:173-185.

Michán, L. y J. Llorente B. 2002. Hacia una historia de la entomología en México. In Biodiversidad, taxonomía y biogeografía de artrópodos de México: hacia una síntesis de su conocimiento. III, J. Llorente B. y J. J. Morrone (eds.), Comisión Nacional para el Conocimiento y Uso de la Biodiversidad / Uiversidad Nacional Autónoma de México, México, D.F. p. 3-52.

Michán, L. y J. Llorente B. 2003. La taxonomía en México durante el siglo XX. Publicaciones Especiales del Museo de Zoología 12: 1-229.

Michán, L., J. Llorente B, A. Luis-Martínez y D. J. Castro. 2004. Breve historia de la taxonomía de Lepidoptera en México durante el S. XX. In Biodiversidad, taxonomía y biogeografía de artrópodos de México: hacia una síntesis de su conocimiento.IV., J. Llorente B, J. J. Morrone y O. YáñezOrdóñez (eds.), Comisión Nacional para el Conocimiento y Uso de la Biodiversidad / Universidad Nacional Autónoma de México, México, D.F. p. 5-42.

**Miller, L. D. y F. M. Brown. 1975. Notes on the rare Mexican pierid Prestonia clarki (Pieridae). Journal of Research on the Lepidoptera, 29:256-258.

Navarro, A. y J. Llorente B. 1994. Museos y la conservación de la biodiversidad. In Taxonomía biológica, J. Llorente B. e I. Luna (comp.), Fondo de Cultura Económica, México, D.F. p. 229-257.

Navarro, A., A. T. Peterson y A. Gordillo-Martínez. 2003a. Museums working together: the atlas of the birds of México. Bulletin B.O.C. 123A:207-224.

Navarro, A., A. T. Peterson, Y. Nakazawa e I. Liebig-Fossas. 2003b. Colecciones biológicas, modelaje de nichos ecológicos y los estudios de la biodiversidad. In Una perspectiva latinoamericana de la biogeografía, J. Morrone y J. Llorente B. (eds.), Comisión Nacional para el Conocimiento y Uso de la Biodiversidad / Universidad Nacional Autónoma de México, México, D.F. p. 115-122.

New, T. R. 1991. Butterfly conservation. Oxford University Press, NewYork. 224 p.

Oñate-Ocaña, L., J. J. Morrone y J. E. Llorente B. 2000. Una evaluación del conocimiento de la distribución de las Papilionidae y Pieridae mexicanas (Insecta: Lepidoptera). Acta Zoologica Mexicana (n.s.) 81:117-132.

**Opler, P. 1986. A new Euchloe (Pieridae) from Northwestern Mexico. Journal of Lepidopterists' Society 40:188-190

**Patterson, D. y J. A. Powell. 1959. Lepidoptera collecting in the sierra San Pedro Mártir, Baja California. Journal of Lepidopterists' Society 13:229-235.

Peláez G., A. 1994. XII. Bases de datos en taxonomía y colecciones científicas. In Taxonomía biológica, J. Llorente B. e I. Luna (comp.), Fondo de Cultura Económica, México, D. F. p. 259-277.

**Pérez R., H. 1977. Distribución geográfica y estructura poblacional de Baronia brevicornis Salv. (Lepidoptera, Papilionidae, Baroniinae) en la República Mexicana. Anales del Instituto de Biología,Universidad Nacional Autónoma de México, Serie Zoología 48:151-164.

**Pérez R., H. y S. Sánchez. 1986. Algunos aspectos demográficos de Baronia brevicornis Salvin en dos localidades de México. Anales del Instituto de Biología, Universidad Nacional Autónoma de México, Serie Zoología 57:191-198.

Peterson, A. T., A. Navarro y H. Benítez. 1998. The need for continued scientific collecting; a geographic analysis of Mexican bird specimens. Ibis 140:288-294.

**Powell, J. A. 1958. Additions to the knowledge of the butterfly fauna of Baja California Norte. Lepidopterists' News 12:26-32.

**Racheli, T. y V. Sbordoni. 1975. A new species of Papilio from Mexico (Lepidoptera, Papilionidae). Fragmenta Entomologica 112:175-183.

**Rau, P. 1942. Clouds of butterflies in Mexico: A study in butterfly aggregations (Lepid: Rhopalocera). Entomological News 53:151-184.

-Reissinger, E., 1972. Zur Taxonomie und Systemaik der Gattung Catasticta Butler (Lepidoptera, Pieridae). Entomologische Zeitschrift 10:97-112, 11:113-124.

**Reyes, C. 1980. Mariposas del suborden Rhopalocera (Lepidoptera) en los alrededores de Aguascalientes, Ags. Tesis, Universidad Autónoma de Aguascalientes, Aguascalientes. $39 \mathrm{p}$.

**Rindge, F. H. 1948. Contributions toward a knowledge of the insect fauna of Lower California 8 Lepidoptera: Rhopalocera. Proceedings of the California Academy of Sciences 24:289-312.

**Rivera, L. T. 1975. Colecta de material entomológico en el estado de Veracruz. Boletín Informativo de la Sociedad Mexicana de Lepidopterología 1:7-8.

Röber, J. 1908-1909. 2. Familie: Pieridae. In Die Gross Schmetterlinge der Erde, A..Seitz (ed.), Kernen, Stuttgart. 5: 53-56, 57-64, 65-72 (1908), 73-80, 81-88, 89-96, 97-104, 105-111 (1909) pls. 18-30.

**Rodríguez, S. 1982. Mariposas del suborden Rhopalocera (Lepidoptera) de Acatlán de Juárez, Jalisco y alrededores. Tesis, Facultad de Ciencias, Universidad Nacional Autónoma de México, México, D.F. 206 p.

**Ross, G. N. 1964a. Life history studies on Mexican butterflies. I. Notes on the earle stages of four Papilionids from Catemaco. Veracruz. Journal of Research on the Lepidoptera 3:9-18.

**Ross, G. N. 1964b. Life history studies on mexican butterflies. III. Nine Rhopalocera from Ocotal Chico, Veracruz. Journal of Research on the Lepidoptera 3:207-229.

**Ross, G. N. 1967. A distributional study of the butterflies of the sierra de Tuxtla in Veracruz, Mexico. Tesis doctorado, Lousiana State University, Baton Rouge. 265 p. 
**Ross, G. N. 1975-1977. An ecological study of the butterflies of the sierra de Tuxtla, Veracruz, México. Journal of Research on the Lepidoptera $14: 103-124 ; 169-188 ; 233-252 ; 15: 41-$ $60 ; 109-128 ; 185-200 ; 225-240 ; 16: 87-130$.

•**Rothschild, W. y K. Jordan. 1906. A revision of American papilios. Novitates Zoologicae 13: 411-752.

**Routledge, E. C. 1977. El suborden Rhopalocera (Lepidoptera) del estado de Tabasco. Su lista, frecuencia, diversidad y distribución. Revista de la Sociedad Mexicana de Lepidopterología 3:57-73.

**Salas, N. 1995. Listado faunístico de la familia Pieridae (Papilionoidea) del estado de Quintana Roo. Tesis, Instituto Tecnológico de Chetumal, Secretaría de Educación Pública, , Chetumal, Quintana Roo. 64 p + 12 pls.

- **Schaus, W. 1898. Two new species of Hesperocharis. Entomological News 9:215

-Schaus, W. 1911. A new Papilio from Florida and one from Mexico (Lepid.). Entomological News 22:438-439.

-Schaus, W. 1920. New species of Lepidoptera in the United States National Museum 57:107-152.

**Seitz, A. 1924. The macrolepidoptera of the world. A systematic description of the hitherto known macrolepidoptera.V. Alfred Kernen, Stuttgart. 1139 p.+ 192 pls.

**Shapiro, A. M. 1981a. A reared gynandromorph of Tatochila (Pieridae). Journal of Research on the Lepidoptera 20:240-242.

**Shapiro, A. M. 1981b. Two homoeotic Pieris rapae of Mexican origin (Pieridae). Journal of Research on the Lepidoptera 20:242-244.

**Shapiro, A. M. y H. Geiger. 1986. Electrophoretic confirmation of the species status of Pontia protodice and Pontia occidentalis (Pieridae). Journal of Research on the Lepidoptera 25:39-47

Smart, P. 1975.The illustrated encyclopedia of butterflies world in colour. Crescent, New York. 275 p.

Soberón, J., J. Llorente B. y L. Oñate-Ocaña. 2000. The use of specimen labels databases for conservation purposes: an example using Mexican papilionid and pierid butterflies. Biodiversity and Conservation 9:1441-1466.

**Spade, T., T. Hamilton y J. W. Brown. 1988. The biology of seven troidine swallowtail butterflies (Papilionidae) in Colima, México. Journal of Research on the Lepidoptera 26:13-26.

Staudinger, O. 1884. Exotische Tagfalter in sysmatischer Reihenfolge mit Berücksichtigung neuer Arten. In Staudinger \& Schatz, Exotische Schmetterlinge 1 (1):3-6, pl. 1-5 (1884); (2):7-10, pl. 6-10 (1884); (3):11-14, pl. 11-15 (1884); (4):1522, pl. 16-20 (1884); (5):23-30, pl. 21-25 (1884); (6):31-38, pl. 26-30 (1884); (7):39-46, pl. 31-35 (1885); (8):47-58, pl. 30-40 (1885); (9):59-82, pl. 41-50 (1885); (10):83-102, pl. 51-60 (1885); (11):103-122, pl. 61-65 (1886); (12):123-138, pl. 66-70 (1886); (13):139-158, pl. 71-75 (1886); (14):159174, pl. 76-80 (1886); (15):175-194, pl. 81-85 (1887); (16):195-214, pl. 86-90 (1887); (17):215-234, pl. 91-95 (1887); (18):235-270, pl. 96-98 (1888); (19):1-2,271-333, pl. 99-100 (1888).

-Stoll, C. 1786-1787-1791. Aangsel van het Werk, de uitlandische Kapellen, voorkomende in de drie Waereld-Deelen
Asia, Africa en America, door den Heere Pieter Cramer, vervattende naauwkeurige afbeeldingen van surinaamsche rupsen en poppen; alsmede van veele zeldzaame en nieuwe ontdekte uitlandische dag- en nagt-kapellen. Nic. Th. Gravius, Amsterdam. i-viii, 1-42, pls. 1-8(1786?-1787); 43184, pls. 9-42(1790).

**Toliver, M. E., R. Holland y S. J. Cary. 1994. Distribution of butterflies in New Mexico (Lepidoptera: Hesperioidea and Papilionoidea). Published by authors. Albuquerque, Nuevo México. 393 p.

Trabulse, E. 1983. Historia de la ciencia en México: estudios y textos, siglo XVI. Fondo de Cultura Económica, México, D.F. 464 p.

**Tyler, H. A. 1975. Swallowtail butterflies. Naturegraph, Healdsburg. 192 p.

Tyler, H., K. S. Brown y K. Wilson. 1994. Swallowtail butterflies of the Americas. A study in biological dynamics, ecological diversity, biosistematics and conservation. Scientific Publishers, Gainesville, Florida. 376 p.

Vargas, I., J. Llorente B. y A. Luis. 1991. Lepidopterofauna de Guerrero. I. Distribución y fenología de los Papilionoidea de la sierra de Atoyac Publicaciones especiales del Museo de Zoología 2. Facultad de Ciencias, Universidad Nacional Autónoma de México, México, D.F. 2:1-127.

Vargas, I., J. Llorente y A. Luis 1994. Listado Lepidopterofaunístico de la sierra de Atoyac de Álvarez en el estado de Guerrero: notas acerca de su distribución local y estacional (Rhopalocera: Papilionoidea). Folia Entomologica Mexicana 86:41-178.

Vargas, I., J. Llorente y A. Luis. 1999. Distribución de los Papilionoidea (Lepidoptera: Rhopalocera) de la sierra de Manantlán (250-1,650 m) en los estados de Jalisco y Colima. Publicaciones Especiales del Museo de Zoología 11, Facultad de Ciencias, Universidad Nacional Autónoma de México, México, D.F. 153 p.

**Vargas, I. F., A. Luis, J. Llorente B. y A. D. Warren. 1996. Butterflies of the state of Jalisco, Mexico. Journal of Lepidopterist's Society 50:97-138

**Vázquez, G. L. 1940. Lepidópteros nuevos de México. Anales del Instituto de Biología， Universidad Nacional Autónoma de México 11:275-284.

**Vázquez, G. L. 1942. Observaciones faunísticas de los Lepidópteros de Izúcar de Matamoros, Puebla. Anales del Instituto de Biología, Universidad Nacional Autónoma de México 13:547-553.

**Vázquez, G. L. 1947. Papilios nuevos de México. Anales del Instituto de Biología Universidad Nacional Autónoma de México 18:249-256.

**Vázquez, G. L. 1949a. Papilios nuevos de México. Anales del Instituto de Biología, Universidad Nacional Autónoma de México 19:233-240.

**Vázquez, G. L. 1949b. Observaciones sobre piéridos mexicanos con descripciones de algunas formas nuevas. Anales del Instituto de Biología Universidad Nacional Autónoma de México 19:469-484.

**Vázquez, G. L. 1951. Observaciones sobre piéridos mexicanos con descripciones de algunas formas nuevas. III. Anales del Instituto de Biología, Universidad Nacional Autónoma 
de México 22:533-555.

**Vázquez, G. L. 1953a. Observaciones sobre piéridos mexicanos con descripciones de algunas formas nuevas V. Anales del Instituto de Biología, Universidad Nacional Autónoma de México 24:435-444.

**Vázquez, G. L. 1953b. Observaciones sobre piéridos mexicanos con descripciones de algunas formas nuevas. IV. Anales del Instituto de Biología Universidad Nacional Autónoma de México 23:257-267.

**Vázquez, G. L. 1953c. Observaciones sobre piéridos mexicanos con descripciones de algunas formas nuevasIII. Anales del Instituto de Biología, Universidad Nacional Autónoma de México 24:171-175.

**Vázquez, G. L. 1954a. Observaciones sobre piéridos mexicanos con descripciones de algunas formas nuevas. VI. Anales del Instituto de Biología, Universidad Nacional Autónoma de México 25:389-390.

**Vázquez, G. L. 1954b. Notas sobre lepidópteros mexicanos. I. Papilionidae y Pieridae de la Mesa de San Diego, Pue., y sus alrededores. Anales del Instituto de Biología Universidad Nacional Autónoma de México 25:391-416.

**Vázquez, G. L. 1954c. Observaciones sobre piéridos mexicanos con descripciones de algunas formas nuevas. V. Las especies del género Itaballia en México. Anales del Instituto de Biología, Universidad Nacional Autónoma de México 24:435-444.

**Vázquez, G. L. 1956. Reconsideración taxonómica de Prestonia clarki Schaus-Phoebis (Prestonia) clarki Schaus-Lepidoptera-Pieridae. Anales del Instituto de Biología Universidad Nacional Autónoma de México 26:477-491.

**Vázquez, G. L. 1957. Papilios nuevos de México. IV. Anales del Instituto de Biología, Universidad Nacional Autónoma de México 27:473-485.

**Vázquez G. L. 1985. Los tipos existentes en la Colección Entomológica del Instituto de Biología, Universidad Nacional Autónoma de México (segundo suplemento) Anales del Instituto de Biología, Universidad Nacional Autónoma de México, Serie Zoología 56:607-640.
**Vázquez, G. L. 1988. Baronia brevicornis Salvin y sus formas (Lepidoptera: Papilionidae: Baroniinae). Anales del Instituto de Biología, Universidad Nacional Autónoma de México, Serie Zoología 58:665-680.

**Vázquez, G. L. 1989. Los tipos existentes en la Colección Entomológica del Instituto de Biología Universidad Nacional Autonoma de México. Cuadernos 2, Instituto de Biología, Universidad Nacional Autónoma de México:1-60.

**Vázquez, G. L. y H. Pérez. 1961. Observaciones sobre la biología de Baronia brevicornis Salv. (Lepidoptera: Papilionidae-Baroniinae). Anales del Instituto de Biología, Universidad Nacional Autónoma de México 32:295-311.

**Vázquez, G. L. y H. Pérez, R. 1967. Nuevas observaciones sobre la biología de Baronia brevicornis Salv. Anales del Instituto de Biología, Universidad Nacional Autónoma de México 37:195-204.

**Vázquez, G. L. y S. Zaragoza. 1979. Tipos existentes en la Colección Entomológica del Instituto de Biología, Universidad Nacional Autónoma de México. Anales del Instituto de Biología, Universidad Nacional Autónoma de México, Serie Zoología 50:575-632

**Velázquez, N. y C. Velázquez. 1975. Viaje de colecta a Jalisco y Colima. Boletín informativo de la Sociedad Mexicana de Lepidopterología 1:6-7.

Warren, A. D., I. Vargas, A. Luis y J. Llorente. 1996. Mariposas diurnas de Jalisco. Dugesiana 3:1-20.

Warren, A. D., I. Vargas, A. Luis y J. Llorente. 1998. Butterflies of the state of Colima, México. Journal of the Lepidopterists' Society 52: 40-72.

**Westwood, J. O. 1834. Description of the nest of a gregarious species of butterfly from Mexico. Transactions of Entomological Society of London 1:38-44.

Whitmore, T. C. y G. T. Prance. 1987. Biogeography and Quaternary history in tropical America. Oxford Monographs on Biogeography 3. Clarendon, Oxford. 214 p.

**Yuste, F., H. Pérez y F. Walls. 1972. Compounds of Papilionid Caterpillars (Baronia brevicornis S.) Experientia 28:11491150. 